\title{
Conversion of 2-Alkylcinnamaldehydes to 2-Alkyindanones Via a Catalytic Intramolecular Friedel-Crafts Reaction
}

\author{
Supporting Information
}

Gary B. Womack*, John G. Angeles, Vincent E. Fanelli and Christie A. Heyer

Firmenich, P.O. Box 5880, Princeton, NJ 08543

gary.womack@fimenich.com

\section{Table of Contents}

1. General Experimental Information

S1

2. Data for 1-Alkoxy-2-Alkyl-1H-Indenes $\mathbf{2 , 5}, \mathbf{6}$, and $\mathbf{7}$

S2-S4

3. Data for 2-Butyl-3-methoxy-1H-indene 18a

S5

4. Data for 2-Alkylindanones $\mathbf{1 9}$

S5-S6

5. References

S6

6. ${ }^{1} \mathrm{H}$ and ${ }^{13} \mathrm{C}$ NMR Spectra of 2

S7-S11

7. ${ }^{1} \mathrm{H}$ and ${ }^{13} \mathrm{C}$ NMR Spectra of 5

$\mathrm{S} 12-\mathrm{S} 13$

9. ${ }^{1} \mathrm{H}$ and ${ }^{13} \mathrm{C}$ NMR Spectra of 6

S14-S18

10. ${ }^{1} \mathrm{H}$ and ${ }^{13} \mathrm{C}$ NMR Spectra of 7

S19-S22

11. ${ }^{1} \mathrm{H}$ and ${ }^{13} \mathrm{C}$ NMR Spectra of $\mathbf{1 8 a}$

$\mathrm{S} 23$

12. ${ }^{1} \mathrm{H}$ and ${ }^{13} \mathrm{C}$ NMR Spectra of $\mathbf{1 9}$

S24-S27

\section{General Experimental Information}

Reagents, anhydrous dichloromethane, anhydrous EtOAc and reagent grade MeOAc (99\%) were used as received from commercial sources. 2-Alkylcinnamaldehydes not commercially available were prepared by aldol condensation of the appropriate arylaldehyde and aldehyde (for typical procedures see reference 1). Reaction progress of indene formation was monitored by $\mathrm{GC}$ analysis after washing an aliquot of the reaction mixture with water or aqueous $10 \% \mathrm{Na}_{2} \mathrm{CO}_{3}$. Column chromatography was performed on $40 \mu \mathrm{m}$ silica gel using hexane and ethyl acetate as eluents. Bulb-to-bulb distillations were performed with a Kugelrohr apparatus, boiling points (bp) corresponding to oven temperatures. NMR spectra were recorded at $400 \mathrm{MHz}$ for ${ }^{1} \mathrm{H}$ and $100 \mathrm{MHz}$ for ${ }^{13} \mathrm{C}$ using $\mathrm{CDCl}_{3}$ as solvent and tetramethylsilane as internal standard. Coupling constants, $J$, are reported in Hertz. IR spectra were taken as neat liquids on $\mathrm{ZnSe}$ plates and reported in wavenumbers $\left(\mathrm{cm}^{-1}\right)$. Mass spectral data were obtained using EI at $70 \mathrm{eV}$ and are reported as $\mathrm{m} / \mathrm{z}$ with intensities relative to the base peak. High-resolution mass spectra were obtained using EI at $70 \mathrm{eV}$. 


\section{1-Methoxy-2-methyl-1H-indene (2a) $)^{2 \mathrm{a}, \mathrm{b}}$}

10 mole $\% \mathrm{FeCl}_{3}$ used; bp $60^{\circ} \mathrm{C}(0.08 \mathrm{mmHg})$, yield $75 \%$

${ }^{1} \mathrm{H}$ NMR $\left(\mathrm{CDCl}_{3}, 400 \mathrm{MHz}\right): \delta 2.03(\mathrm{~s}, 3 \mathrm{H}), 3.03(\mathrm{~s}, 3 \mathrm{H}), 4.85(\mathrm{~s}, 1 \mathrm{H}), 6.44(\mathrm{~s}, 1 \mathrm{H})$, $7.11(\mathrm{t}, J=7.4,1 \mathrm{H}), 7.12(\mathrm{~d}, J=7.2,1 \mathrm{H}), 7.22(\mathrm{t}, J=7.4,1 \mathrm{H}), 7.41(\mathrm{~d}, J=7.2 \mathrm{~Hz}, 1 \mathrm{H})$;

${ }^{13} \mathrm{C} \mathrm{NMR}\left(\mathrm{CDCl}_{3}, 100 \mathrm{MHz}\right): \delta 14.1(\mathrm{q}), 51.8(\mathrm{q}), 84.9$ (d), 120.1 (d), 123.7 (d), 124.6 (d), 128.4 (d), 128.7 (d), 141.8 (s), 143.9 (s), 145.8 (s); IR: 3048, 2935, 2823, 1625, 1606, 1462, 1441, 1320, 1207, 1105, 1079, 944, $898 \mathrm{~cm}^{-1}$; MS: 160(M $\left.\mathrm{M}^{+}, 78\right)$, 145(100), 129 (26), 128(46), 117(36), 115(56); HRMS calcd for $\mathrm{C}_{11} \mathrm{H}_{12} \mathrm{O} 160.0888$, found 160.0884 .

\section{1-Methoxy-2-ethyl-1H-indene $(2 \mathrm{~b})^{2 \mathrm{a}}$} bp $55-75^{\circ} \mathrm{C}(0.018 \mathrm{mmHg})$, yield $93 \%$

${ }^{1} \mathrm{H}$ NMR $\left(\mathrm{CDCl}_{3}, 400 \mathrm{MHz}\right): \delta 1.22(\mathrm{t}, J=7.4,3 \mathrm{H}), 2.29-2.48(\mathrm{~m}, 2 \mathrm{H}), 3.02(\mathrm{~s}, 3 \mathrm{H})$, $4.93(\mathrm{~s}, 1 \mathrm{H}), 6.43(\mathrm{~s}, 1 \mathrm{H}), 7.11(\mathrm{t}, J=7.2,1 \mathrm{H}), 7.14(\mathrm{~d}, J=7.2,1 \mathrm{H}), 7.22(\mathrm{t}, J=7.4,1 \mathrm{H})$, $7.41(\mathrm{~d}, J=7.4,1 \mathrm{H}) ;{ }^{13} \mathrm{C} \mathrm{NMR}\left(\mathrm{CDCl}_{3}, 100 \mathrm{MHz}\right): \delta 12.4(\mathrm{q}), 21.6(\mathrm{t}), 51.9(\mathrm{q}), 83.9$ (d), 120.3 (d), 123.8 (d), 124.7 (d), 126.7 (d), 128.4 (d), 141.9 (s), 143.8 (s), 152.2 (s); MS: 174(M+ $\left.\mathrm{M}^{+}, 57\right), 159$ (100), 145 (80), 141 (16), 131(20), 128 (46), 115 (44) 102 (11), 91 (18); HRMS calculated for $\mathrm{C}_{12} \mathrm{H}_{14} \mathrm{O}$ 174.1045, found 174.1048.

\section{1-Methoxy-2-propyl-1H-indene (2c)}

bp $80-90^{\circ} \mathrm{C}(0.018 \mathrm{mmHg})$, yield $92 \%$

${ }^{1} \mathrm{H}$ NMR $\left(\mathrm{CDCl}_{3}, 400 \mathrm{MHz}\right): \delta 0.99(\mathrm{t}, J=7.2,3 \mathrm{H}), 1.54-1.76(\mathrm{~m}, 2 \mathrm{H}), 2.34(\mathrm{t}, J=7.7$, $2 \mathrm{H}), 3.02(\mathrm{~s}, 3 \mathrm{H}), 4.93(\mathrm{~s}, 1 \mathrm{H}), 6.44(\mathrm{~s}, 1 \mathrm{H}), 7.11(\mathrm{t}, J=7.4,1 \mathrm{H}), 7.14(\mathrm{~d}, J=7.2,1 \mathrm{H})$, $7.22(\mathrm{t}, J=7.4,1 \mathrm{H}), 7.42(\mathrm{~d}, J=7.2,1 \mathrm{H}) ;{ }^{13} \mathrm{C} \mathrm{NMR}\left(\mathrm{CDCl}_{3}, 100 \mathrm{MHz}\right): \delta 14.1(\mathrm{q})$, $21.5(\mathrm{t}), 30.5$ (t), 51.9 (q), 83.8 (d), 120.3 (d), 123.8 (d), 124.7 (d), 127.6 (d), 128.4 (d), 141.9 (s), 143.8 (s), 150.5 (s); MS: 188( M $\left.^{+}, 19\right), 159$ (100), 145 (19), 141 (6), 129 (22), 128 (27), 115 (22), 102 (6), 91 (8); HRMS calcd for $\mathrm{C}_{13} \mathrm{H}_{16} \mathrm{O}$ 188.1201, found 188.1198 .

\section{1-Methoxy-2-butyl-1H-indene (2d)}

bp $85-90^{\circ} \mathrm{C}(0.019 \mathrm{mmHg})$, yield $95 \%$

${ }^{1} \mathrm{H} \mathrm{NMR}\left(\mathrm{CDCl}_{3}, 400 \mathrm{MHz}\right): \delta 0.95(\mathrm{t}, J=7.2,3 \mathrm{H}), 1.36-1.46(\mathrm{~m}, 2 \mathrm{H}), 1.51-1.70(\mathrm{~m}$, $2 \mathrm{H}), 2.29-2.43(\mathrm{~m}, 2 \mathrm{H}), 3.02(\mathrm{~s}, 3 \mathrm{H}), 4.93(\mathrm{~s}, 1 \mathrm{H}), 6.43(\mathrm{~s}, 1 \mathrm{H}), 7.11(\mathrm{t}, J=7.2,1 \mathrm{H})$, $7.14(\mathrm{~d}, J=7.2,1 \mathrm{H}), 7.22(\mathrm{t}, J=7.2,1 \mathrm{H}), 7.41(\mathrm{~d}, J=7.2,1 \mathrm{H}) ;{ }^{13} \mathrm{C} \mathrm{NMR}\left(\mathrm{CDCl}_{3}, 100\right.$ $\mathrm{MHz}): \delta 14.0(\mathrm{q}), 22.7(\mathrm{t}), 28.1(\mathrm{t}), 30.4(\mathrm{t}), 51.8(\mathrm{q}), 83.8(\mathrm{~d}), 120.3(\mathrm{~d}), 123.8(\mathrm{~d})$, 124.6 (d), 127.4 (d), 128.4 (d), 141.8 (s), 143.8 (s), 150.7 (s); IR: 2958, 2931, 1618, 1464, 1321, 1202, 1106, 1079, 948, $898 \mathrm{~cm}^{-1}$; MS: 202 (M+1 17$), 159$ (100), 145 (18), 141 (5), 129 (19), 128 (21), 115 (15), 102 (4), 91 (5); HRMS calcd for $\mathrm{C}_{14} \mathrm{H}_{18} \mathrm{O}$ 202.1358, found 202.1356 .

\section{1-Methoxy-2-pentyl-1H-indene $(2 \mathrm{e})^{3}$}

bp $95-100^{\circ} \mathrm{C}(0.024 \mathrm{mmHg})$, yield $90 \%$

${ }^{1} \mathrm{H}$ NMR $\left(\mathrm{CDCl}_{3}, 400 \mathrm{MHz}\right): \delta 0.91(\mathrm{t}, J=7.2,3 \mathrm{H}), 1.30-1.42(\mathrm{~m}, 4 \mathrm{H}), 1.52-1.72(\mathrm{~m}$, $2 \mathrm{H}), 2.28-2.42(\mathrm{~m}, 2 \mathrm{H}), 3.01(\mathrm{~s}, 3 \mathrm{H}), 4.92(\mathrm{~s}, 1 \mathrm{H}), 6.43(\mathrm{~s}, 1 \mathrm{H}), 7.11(\mathrm{t}, J=7.2,1 \mathrm{H})$, $7.13(\mathrm{~d}, J=7.2,1 \mathrm{H}), 7.21(\mathrm{t}, J=7.2,1 \mathrm{H}), 7.41(\mathrm{~d}, J=7.2,1 \mathrm{H}) ;{ }^{13} \mathrm{C} \mathrm{NMR}\left(\mathrm{CDCl}_{3}, 100\right.$ MHz): $\delta 14.1(\mathrm{q}), 22.6(\mathrm{t}), 28.0(\mathrm{t}), 28.4(\mathrm{t}), 31.8(\mathrm{t}), 51.9(\mathrm{q}), 83.8(\mathrm{~d}), 120.3(\mathrm{~d})$, 123.8 (d), 124.6 (d), 127.5 (d), 128.4 (d), 141.8 (s), 143.8 (s), 150.7 (s); IR: 2958, 2930, 1618, 1464, 1321, 1202, 1106, 1079, 947, $898 \mathrm{~cm}^{-1}$; MS: 216(M+1 16$), 159$ (100), 145 (15), 129 (16), 128 (18), 115 (12); HRMS calcd for $\mathrm{C}_{15} \mathrm{H}_{20} \mathrm{O} 216.1514$, found 216.1511 . 


\section{1-Ethoxy-2-methyl-1H-indene (5a)}

$15 \mathrm{~mol} \% \mathrm{FeCl}_{3}$, EtOAc, $70^{\circ} \mathrm{C}, 7 \mathrm{~h}$; bp $60-80^{\circ} \mathrm{C}(0.045 \mathrm{mmHg})$, yield $69 \%$

${ }^{1} \mathrm{H}$ NMR $\left(\mathrm{CDCl}_{3}, 400 \mathrm{MHz}\right): \delta 1.16(\mathrm{t}, J=7.2,3 \mathrm{H}), 2.03(\mathrm{~s}, 3 \mathrm{H}), 3.16-3.28(\mathrm{~m}, 2 \mathrm{H})$, $4.87(\mathrm{~s}, 1 \mathrm{H}), 6.40(\mathrm{~s}, 1 \mathrm{H}), 7.10(\mathrm{t}, J=7.4,1 \mathrm{H}), 7.11(\mathrm{~d}, J=7.2,1 \mathrm{H}), 7.20(\mathrm{t}, J=7.4,1 \mathrm{H})$, $7.42(\mathrm{~d}, J=7.2,1 \mathrm{H}) ;{ }^{13} \mathrm{C} \mathrm{NMR}\left(\mathrm{CDCl}_{3}, 100 \mathrm{MHz}\right): \delta 14.1(\mathrm{q}), 15.7(\mathrm{q}), 60.0(\mathrm{t}), 84.5$ (d), 120.1 (d), 123.6 (d), 124.6 (d), 128.1 (d), 128.2 (d), 142.6 (s), 143.6 (s), 146.4 (s); IR: 2978, 2883, 1637, 1344, 1312, 1175, 1136, $1037 \mathrm{~cm}^{-1}$; MS: $174\left(\mathrm{M}^{+}, 99\right), 146$ (62), 145 (98), 131 (100), 128 (67), 117 (56), 115 (65); HRMS calcd for $\mathrm{C}_{12} \mathrm{H}_{14} \mathrm{O}$ 174.1045, found 174.1042.

\section{1-Ethoxy-2-butyl-1H-indene (5b)}

$5 \mathrm{~mol} \% \mathrm{FeCl}_{3}, \mathrm{DCM}$, reflux, $4 \mathrm{~h}$; bp $110-120^{\circ} \mathrm{C}(0.018 \mathrm{mmHg})$, yield $96 \%$

${ }^{1} \mathrm{H} \mathrm{NMR}\left(\mathrm{CDCl}_{3}, 400 \mathrm{MHz}\right): \delta 0.95(\mathrm{t}, J=7.4,3 \mathrm{H}), 1.15(\mathrm{t}, J=6.9,3 \mathrm{H}), 1.36-1.46(\mathrm{~m}$, $2 \mathrm{H}), 1.50-1.70(\mathrm{~m}, 2 \mathrm{H}), 2.30-2.45(\mathrm{~m}, 2 \mathrm{H}), 3.15-3.27(\mathrm{~m}, 2 \mathrm{H}), 4.95(\mathrm{~s}, 1 \mathrm{H}), 6.41(\mathrm{~s}$, $1 \mathrm{H}), 7.10(\mathrm{t}, J=7.2,1 \mathrm{H}), 7.13(\mathrm{~d}, J=7.2,1 \mathrm{H}), 7.21(\mathrm{t}, J=7.2,1 \mathrm{H}), 7.42(\mathrm{~d}, J=7.2,1 \mathrm{H})$; ${ }^{13} \mathrm{C} \mathrm{NMR}\left(\mathrm{CDCl}_{3}, 100 \mathrm{MHz}\right): \delta 14.0(\mathrm{q}), 15.7(\mathrm{q}), 22.7(\mathrm{t}), 28.1(\mathrm{t}), 30.5(\mathrm{t}), 60.0(\mathrm{t})$, 83.4 (d), 120.2 (d), 123.7 (d), 124.6 (d), 126.9 (d), 128.2 (d), 142.5 (s), 143.6 (s), 151.3 (s); IR: 2960, 2929, 2873, 1618, 1462, 1200, 1171, 1106, 1078, 1017, $910 \mathrm{~cm}^{-}$ 1; MS: $216\left(\mathrm{M}^{+}, 33\right), 173$ (85), 145 (100), 131 (29), 129 (30), 128 (32), 117 (14), 115 (26); HRMS calcd for $\mathrm{C}_{15} \mathrm{H}_{20} \mathrm{O} 216.1514$, found 216.1504 .

\section{1-Methoxy-2,6-dimethyl-1H-indene $(6 \mathrm{a})^{2 \mathrm{a}}$}

bp $60-70^{\circ} \mathrm{C}(0.020 \mathrm{mmHg})$, yield $67 \%$

${ }^{1} \mathrm{H} \mathrm{NMR}\left(\mathrm{CDCl}_{3}, 400 \mathrm{MHz}\right): \delta 2.00(\mathrm{~s}, 3 \mathrm{H}), 2.35$ (s, 3H), $3.03(\mathrm{~s}, 3 \mathrm{H}), 4.82(\mathrm{~s}, 1 \mathrm{H})$, $6.40(\mathrm{~s}, 3 \mathrm{H}), 6.99-7.04(\mathrm{~m}, 2 \mathrm{H}), 7.24(\mathrm{~s}, 1 \mathrm{H}) ;{ }^{13} \mathrm{C} \mathrm{NMR}\left(\mathrm{CDCl}_{3}, 100 \mathrm{MHz}\right): \delta 14.1$ (q), 21.4 (q), 51.8 (q), 84.8 (d), 119.8 (d), 124.8 (d), 128.5 (d), 128.8 (d), 134.3 (s), 141.2 (s), 142.1 (s), 144.7 (s); MS: 174 (M+, 85), 159 (100), 144 (12), 143 (13), 141 (13), 131 (24), 128 (23), 115 (24), 91 (11).

\section{1-Methoxy-2,4-dimethyl-1H-indene $(6 \mathrm{~b})^{2 \mathrm{a}}$}

bp $60-67^{\circ} \mathrm{C}(0.018 \mathrm{mmHg})$, yield $83 \%$

${ }^{1} \mathrm{H}$ NMR $\left(\mathrm{CDCl}_{3}, 400 \mathrm{MHz}\right): \delta 2.03(\mathrm{~s}, 3 \mathrm{H}), 2.32(\mathrm{~s}, 3 \mathrm{H}), 3.02(\mathrm{~s}, 3 \mathrm{H}), 4.84(\mathrm{~s}, 1 \mathrm{H})$, $6.54(\mathrm{~s}, 1 \mathrm{H}), 7.02(\mathrm{~d}, J=4.6,2 \mathrm{H}), 7.24(\mathrm{t}, J=4.6,1 \mathrm{H}) ;{ }^{13} \mathrm{C} \mathrm{NMR}\left(\mathrm{CDCl}_{3}, 100 \mathrm{MHz}\right): \delta$ 14.2 (q), 18.0 (q), 51.7 (q), 85.1 (q), 121.1 (d), 124.7 (d), 126.8 (d), 129.2 (s), 129.6 (d), 141.7 (s), 142.3 (s), 145.2 (s); MS: $174\left(\mathrm{M}^{+}, 69\right), 159$ (100), 144( 12), 143 (14), 141 (14), 131 (23), 128 (27), 116 (17), 115 (29), 91 (15); HRMS calcd for $\mathrm{C}_{12} \mathrm{H}_{14} \mathrm{O}$ 174.1045, found 174.1042.

\section{1,4-Dimethoxy-2-methyl-1H-indene (6d)}

bp $76-87^{\circ} \mathrm{C}(0.018 \mathrm{mmHg})$, yield $56 \%$, recrystallized from pentane, $\mathrm{mp}=64-65^{\circ} \mathrm{C}$ ${ }^{1} \mathrm{H} \mathrm{NMR}\left(\mathrm{CDCl}_{3}, 400 \mathrm{MHz}\right): \delta 2.01(\mathrm{~s}, 3 \mathrm{H}), 3.01(\mathrm{~s}, 3 \mathrm{H}), 3.83(\mathrm{~s}, 3 \mathrm{H}), 4.85(\mathrm{~s}, 1 \mathrm{H})$, $6.60(\mathrm{~s}, 1 \mathrm{H}), 6.78(\mathrm{~d}, J=7.7,1 \mathrm{H}), 7.05-7.12(\mathrm{~m}, 2 \mathrm{H}) ;{ }^{13} \mathrm{C} \mathrm{NMR}\left(\mathrm{CDCl}_{3}, 100 \mathrm{MHz}\right): \delta$ 14.0 (q), 51.7 (q), 55.4 (q), 85.3 (d), 111.1 (d), 116.8 (d), 124.7 (d), 126.0 (d), 131.8 (s), 143.8 (s), 143.9 (s), 152.2 (s); MS: $190\left(\mathrm{M}^{+}, 71\right), 175$ (100), 160 (17), 159 (16), 147 (14), 145 (9), 129 (17), 115 (35); HRMS calcd for $\mathrm{C}_{12} \mathrm{H}_{14} \mathrm{O}_{2}$ 190.0994, found 190.0984 .

\section{6-Isopropyl-1-methoxy-2-methyl-1H-indene (6e)}

bp $85-92^{\circ} \mathrm{C}$ (0.018 mTorr), yield $72 \%$ 
${ }^{1} \mathrm{H}$ NMR $\left(\mathrm{CDCl}_{3}, 400 \mathrm{MHz}\right): \delta 1.25(\mathrm{~d}, J=6.9,6 \mathrm{H}), 2.01$ (s, 3H), 2.90 (septet, $J=6.9$, $1 \mathrm{H}), 3.03(\mathrm{~s}, 3 \mathrm{H}), 4.84(\mathrm{~s}, 1 \mathrm{H}), 6.40(\mathrm{~s}, 1 \mathrm{H}), 7.04(\mathrm{~d}, J=7.7,1 \mathrm{H}), 7.08(\mathrm{dd}, J=1.5$ and 7.7, 1H), $7.31(\mathrm{~s}, 1 \mathrm{H}) ;{ }^{13} \mathrm{C} \mathrm{NMR}\left(\mathrm{CDCl}_{3}, 100 \mathrm{MHz}\right): \delta 14.1(\mathrm{q}), 24.2(\mathrm{q}), 24.3(\mathrm{q})$, 34.1 (d), 51.8 (q), 84.9 (d), 119.8 (d), 122.1 (d), 126.3 (d), 128.5 (d), 141.6 (s), 142.1 (s), 144.9 (s), 145.6 (s); MS: $202\left(\mathrm{M}^{+}, 58\right), 187$ (60), 172 (12), 160 (14), 159 (100), 157 (12), 156 (14), 144 (10), 141 (15), 128 (24), 117 (12), 115 (23); HRMS calcd for $\mathrm{C}_{14} \mathrm{H}_{18} \mathrm{O} 202.1358$, found 202.1358 .

\section{6-tert-Butyl-1-methoxy-2-methyl-1H-indene (6f)}

flash chromatographed (hexane/EtOAc $98: 2)$, bp $90-100^{\circ} \mathrm{C}(0.020 \mathrm{mmHg})$, yield $65 \%$ ${ }^{1} \mathrm{H} \mathrm{NMR}\left(\mathrm{CDCl}_{3}, 400 \mathrm{MHz}\right): \delta 1.33$ (s, 9H), $2.02(\mathrm{~s}, 3 \mathrm{H}), 3.02(\mathrm{~s}, 3 \mathrm{H}), 4.85(\mathrm{~s}, 1 \mathrm{H})$, $6.41(\mathrm{~s}, 1 \mathrm{H}), 7.05(\mathrm{~d}, J=7.7,1 \mathrm{H}), 7.25(\mathrm{dd}, J=1.5$ and $7.7,1 \mathrm{H}), 7.48(\mathrm{~s}, 1 \mathrm{H}) ;{ }^{13} \mathrm{C}$ NMR ( $\left.\mathrm{CDCl}_{3}, 100 \mathrm{MHz}\right): \delta 14.1$ (q), $31.6(\mathrm{q}), 34.6$ (s), 51.7 (q), 85.0 (d), 119.5 (d), 121.1 (d), 125.0 (d), 128.4 (d), 141.2 (s), 141.7 (s), 145.1 (s), 147.9 (s); MS: $216\left(\mathrm{M}^{+}\right.$, 76), 201 (100), 186 (21), 170 (18), 159 (99), 128 (21), 115 (19); HRMS calcd for $\mathrm{C}_{15} \mathrm{H}_{20} \mathrm{O} 216.1514$, found 216.1502.

\section{1-Methoxy-2-ethyl-6-methyl-1H-indene (7a)}

bp $80-90^{\circ} \mathrm{C}(0.02 \mathrm{mmHg})$, yield $92 \%$

${ }^{1} \mathrm{H} \mathrm{NMR}\left(\mathrm{CDCl}_{3}, 400 \mathrm{MHz}\right): \delta 1.21(\mathrm{t}, J=7.4,3 \mathrm{H}), 2.35(\mathrm{~s}, 3 \mathrm{H}), 2.27-2.46(\mathrm{~m}, 2 \mathrm{H})$, $3.02(\mathrm{~s}, 3 \mathrm{H}), 4.91(\mathrm{~s}, 1 \mathrm{H}), 6.40(\mathrm{~s}, 1 \mathrm{H}), 7.02(\mathrm{~s}, 2 \mathrm{H}), 7.24, \mathrm{~s} 1 \mathrm{H}) ;{ }^{13} \mathrm{C} \mathrm{NMR}\left(\mathrm{CDCl}_{3}\right.$, $100 \mathrm{MHz}): \delta 12.5(\mathrm{q}), 21.4(\mathrm{q}), 21.5(\mathrm{t}), 51.8(\mathrm{q}), 83.8(\mathrm{~d}), 119.9(\mathrm{~d}), 124.9(\mathrm{~d}), 126.6$ (d), 128.8 (d), 134.3 (s), 141.1 (s), 142.1 (s), 151.0 (s); MS: $188\left(\mathrm{M}^{+}, 72\right), 173$ (100), 159 (69), 145 (20), 141 (21), 128, (17), 115 (18); HRMS calcd for $\mathrm{C}_{13} \mathrm{H}_{16} \mathrm{O}$ 188.1201, found 188.1197 .

\section{1-Methoxy-2-ethyl-4-methyl-1H-indene (7b)}

bp $65-72^{\circ} \mathrm{C}(0.018 \mathrm{mmHg})$, yield $96 \%$

${ }^{1} \mathrm{H}$ NMR $\left(\mathrm{CDCl}_{3}, 400 \mathrm{MHz}\right): \delta 1.24(\mathrm{t}, J=7.4,3 \mathrm{H}), 2.34(\mathrm{~s}, 3 \mathrm{H}), 2.50-2.30(\mathrm{~m}, 2 \mathrm{H})$, $3.01(\mathrm{~s}, 3 \mathrm{H}), 4.93(\mathrm{~s}, 1 \mathrm{H}), 6.54(\mathrm{~s}, 1 \mathrm{H}), 7.03$ (overlapping d, $J=3.6$ and 5.1, 2H), 7.25 $(\mathrm{dd}, J=3.6$ and $5.1,1 \mathrm{H}) ;{ }^{13} \mathrm{C} \mathrm{NMR}\left(\mathrm{CDCl}_{3}, 100 \mathrm{MHz}\right): \delta 12.5(\mathrm{q}), 18.0(\mathrm{q}), 21.7(\mathrm{t})$, 51.8 (q), 84.1 (d), 121.3 (d), 124.7 (d), 124.8 (d), 129.4 (s), 129.6 (d), 141.7 (s), 142.3 (s), 151.5 (s); MS: $188\left(\mathrm{M}^{+}, 56\right), 173$ (93), 159 (100), 145 (19), 142 (23), 141 (31), 129 (21), 128 (30), 115 (41); HRMS calcd for $\mathrm{C}_{13} \mathrm{H}_{16} \mathrm{O} 188.1201$, found 188.1192 .

\section{2-Ethyl-1,6-dimethoxy-1H-indene (7c)}

bp $95-100^{\circ} \mathrm{C}(0.018 \mathrm{mmHg})$, yield $82 \%$

${ }^{1} \mathrm{H} \mathrm{NMR}\left(\mathrm{CDCl}_{3}, 400 \mathrm{MHz}\right): \delta 1.21(\mathrm{t}, J=7.4,3 \mathrm{H}), 2.26-2.45(\mathrm{~m}, 2 \mathrm{H}), 3.01(\mathrm{~s}, 3 \mathrm{H})$, $3.80(\mathrm{~s}, 3 \mathrm{H}), 4.91(\mathrm{~s}, 1 \mathrm{H}), 6.38(\mathrm{~s}, 1 \mathrm{H}), 6.76\left(\mathrm{dd}, J=8.2, J_{4}=2.6,1 \mathrm{H}\right), 7.04(\mathrm{~d}, J=8.2$, $1 \mathrm{H}), 7.05$ (broad s, $1 \mathrm{H}) ;{ }^{13} \mathrm{C} \mathrm{NMR}\left(\mathrm{CDCl}_{3}, 100 \mathrm{MHz}\right): \delta 12.5(\mathrm{q}), 21.5(\mathrm{t}), 51.7(\mathrm{q})$, 55.5 (q), 83.8 (d), 111.1 (d), 113.0 (d), 120.5 (d), 126.3 (d), 136.7 (s), 143.8 (s), 149.9 (s), 158.0 (s); MS: 204 (M+ $\left.\mathrm{M}^{+}, 73\right), 189$ (100), 175 (56), 161 (30), 160 (29), 158 (16), 128 (17), 115 (29); HRMS calcd for $\mathrm{C}_{13} \mathrm{H}_{16} \mathrm{O}_{2}$ 204.1150, found 204.1156.

\section{2-Ethyl-1,4-dimethoxy-1H-indene (7d)}

bp $80-100^{\circ} \mathrm{C}(0.018 \mathrm{mmHg})$, yield $80 \%$ 
${ }^{1} \mathrm{H} \mathrm{NMR}\left(\mathrm{CDCl}_{3}, 400 \mathrm{MHz}\right): \delta 1.22(\mathrm{t}, J=7.4,3 \mathrm{H}), 2.27-2.48(\mathrm{~m}, 2 \mathrm{H}), 3.00(\mathrm{~s}, 3 \mathrm{H})$, $3.85(\mathrm{~s}, 3 \mathrm{H}), 4.94(\mathrm{~s}, 1 \mathrm{H}), 6.61(\mathrm{~s}, 1 \mathrm{H}), 6.79(\mathrm{~d}, J=7.7,1 \mathrm{H}), 7.06-7.12(\mathrm{~m}, 2 \mathrm{H}) ;{ }^{13} \mathrm{C}$ NMR ( $\left.\mathrm{CDCl}_{3}, 100 \mathrm{MHz}\right): \delta 12.4(\mathrm{q}), 21.6(\mathrm{t}), 51.8(\mathrm{q}), 55.4(\mathrm{q}), 84.3(\mathrm{~d}), 110.9(\mathrm{~d})$, 116.8 (d), 122.7 (d), 126.1 (d), 131.7 (s), 143.8 (s), 150.3 (s), 152.2 (s); MS: $204\left(\mathrm{M}^{+}\right.$, 62), 189 (75), 175 (100), 161 (17), 160 (17), 145 (13), 128 (20), 115 (29); HRMS calcd for $\mathrm{C}_{13} \mathrm{H}_{16} \mathrm{O}_{2} 204.1150$, found 204.1147.

\section{2-Butyl-3-methoxy-1H-indene (18a)}

2d $(25.3 \mathrm{~g}, 0.125 \mathrm{~m})$ and triethyl amine $(25.3 \mathrm{~g}, 0.25 \mathrm{~m})$ were mixed in $150 \mathrm{~mL}$ of ethyl acetate for three days. The mixture was concentrated to remove solvent and subjected to bulb-to-bulb distillation $\left(50-60^{\circ} \mathrm{C}\right.$ oven temperature, 3 mbar) to remove triethyl amine. Further distillation $\left(102-105^{\circ} \mathrm{C}, 0.020 \mathrm{mmHg}\right)$ yielded $24.8 \mathrm{~g}$ of $\mathbf{1 8 a}$ $(0.123 \mathrm{~m}, 98 \%$ yield $)$ as a pale yellow oil.

${ }^{1} \mathrm{H} \mathrm{NMR}\left(\mathrm{CDCl}_{3}, 400 \mathrm{MHz}\right): \delta 0.93(\mathrm{t}, J=7.2,3 \mathrm{H}), 1.37$ (sextet, $\left.J=7.2,2 \mathrm{H}\right), 1.49-1.56$ $(\mathrm{m}, 2 \mathrm{H}), 2.48(\mathrm{t}, J=7.7,2 \mathrm{H}), 3.20(\mathrm{~s}, 2 \mathrm{H}), 3.89(\mathrm{~s}, 3 \mathrm{H}), 7.13(\mathrm{t}, J=7.7,1 \mathrm{H}), 7.25(\mathrm{t}$, $J=7.7,1 \mathrm{H}), 7.33(\mathrm{t}, J=7.7,2 \mathrm{H}) ;{ }^{13} \mathrm{C} \mathrm{NMR}\left(\mathrm{CDCl}_{3}, 100 \mathrm{MHz}\right): \delta 14.0(\mathrm{q}), 22.7(\mathrm{t})$, $26.4(\mathrm{t}), 31.8$ (t), 36.7 (t), 59.7 (q), 117.6 (d), 123.8 (d), 124.2 (d), 126.1 (d), 127.6 (s), 141.1 (s), 141.2 (s), 152.6 (s); IR: 2958, 2932, 1632, 1465, 1356, 1301, 1133, 990 $\mathrm{cm}^{-1}$; MS: $202\left(\mathrm{M}^{+}, 20\right), 160$ (14), 159 (100), 145 (11), 131 (6), 129 (7), 128 (9), 115 (18), 91 (8); HRMS calcd for $\mathrm{C}_{14} \mathrm{H}_{18} \mathrm{O} 202.1358$, found 202.1361 .

\section{2-Butylindanone (19a)}

bp $120^{\circ} \mathrm{C}(0.02 \mathrm{mmHg})$, yield $95 \%$ from $2 d$. Spectral data matched that reported in the literature. ${ }^{4}$

${ }^{1} \mathrm{H}$ NMR $\left(\mathrm{CDCl}_{3}, 400 \mathrm{MHz}\right): \delta 0.92(\mathrm{t}, J=7.2,3 \mathrm{H}), 1.30-1.50(\mathrm{~m}, 5 \mathrm{H}), 1.90-2.00(\mathrm{~m}$, $1 \mathrm{H}), 2.61-2.67(\mathrm{~m}, 1 \mathrm{H}), 2.81(\mathrm{dd}, J=17.1,3.8,1 \mathrm{H}), 3.31(\mathrm{dd}, J=17.1,7.9,1 \mathrm{H}), 7.34$ (t, $J=7.6,1 \mathrm{H}), 7.44(\mathrm{~d}, J=7.6,1 \mathrm{H}), 7.57(\mathrm{t}, J=7.6,1 \mathrm{H}), 7.74(\mathrm{~d}, J=7.6,1 \mathrm{H}) ;{ }^{13} \mathrm{C}$ NMR $\left(\mathrm{CDCl}_{3}, 100 \mathrm{MHz}\right): \delta 14.0(\mathrm{q}), 22.7(\mathrm{t}), 29.6(\mathrm{t}), 31.2(\mathrm{t}), 32.9(\mathrm{t}), 47.4(\mathrm{~d}), 123.8(\mathrm{~d})$, 126.6 (d), 127.3 (d), 134.6 (d), 136.9 (s), 153.8 (s), 209.1 (s); IR: 2958, 2931, 2858, 1713, 1610, 1465, $1287 \mathrm{~cm}^{-1}$; MS: 188 (2), 145 (13), 133 (10), 132 (100), 131 (16), 115 (11); HRMS calcd for $\mathrm{C}_{13} \mathrm{H}_{16} \mathrm{O}$ 188.1201, found 188.1209 .

\section{2-Pentylindanone $(19 \mathrm{~b})^{5}$}

bp $102-115^{\circ} \mathrm{C}(0.025 \mathrm{mmHg})$, yield $91 \%$ from $\mathbf{2 e}$

${ }^{1} \mathrm{H}$ NMR $\left(\mathrm{CDCl}_{3}, 400 \mathrm{MHz}\right): \delta 0.89(\mathrm{t}, J=6.9,3 \mathrm{H}), 1.28-1.36(\mathrm{~m}, 3 \mathrm{H}), 1.38-1.50(\mathrm{~m}$, $4 \mathrm{H}), 1.89-2.00(\mathrm{~m}, 1 \mathrm{H}), 2.61-2.68(\mathrm{~m}, 1 \mathrm{H}), 2.80(\mathrm{dd}, J=17.2,4.1,1 \mathrm{H}), 3.31(\mathrm{dd}$, $J=17.2,7.7,1 \mathrm{H}), 7.35(\mathrm{t}, J=7.6,1 \mathrm{H}), 7.44(\mathrm{~d}, 7.6,1 \mathrm{H}), 7.56(\mathrm{t}, J=7.6,1 \mathrm{H}), 7.73(\mathrm{~d}$, $J=7.6,1 \mathrm{H}) ;{ }^{13} \mathrm{C} \mathrm{NMR}\left(\mathrm{CDCl}_{3}, 100 \mathrm{MHz}\right): \delta 14.0(\mathrm{q}), 22.5(\mathrm{t}), 27.1(\mathrm{t}), 31.4(\mathrm{t}), 31.8$ (t), $32.9(\mathrm{t}), 47.5$ (d), 123.8 (d), 126.5 (d), 127.3 (d), 134.6 (d), 136.9 (s), 153.8 (s), 209.0 (s); IR: 2958, 2930, 2856, 1712, 1610, $1464 \mathrm{~cm}^{-1}$; MS: $202\left(\mathrm{M}^{+}, 2\right), 145(16)$, 133 (11), 132 (100), 131 (13), 115 (10). HRMS calcd for $\mathrm{C}_{14} \mathrm{H}_{18} \mathrm{O} 202.1358$, found 202.1355; Anal. calcd for $\mathrm{C}_{14} \mathrm{H}_{18} \mathrm{O}$ : C 83.12, H 8.97; found $\mathrm{C} 82.80$, 8.77.

\section{2,4-Dimethylindan-1-one (19c)}

bp $100-107^{\circ} \mathrm{C}(0.025 \mathrm{mmHg})$, yield $94 \%$ from $\mathbf{6 b}, \mathrm{mp}=34-35^{\circ} \mathrm{C}$ (lit. $\left.{ }^{6} 36-37^{\circ} \mathrm{C}\right)$ ${ }^{1} \mathrm{H}$ NMR $\left(\mathrm{CDCl}_{3}, 400 \mathrm{MHz}\right): \delta 1.31(\mathrm{~d}, J=7.2,3 \mathrm{H}), 2.34(\mathrm{~s}, 3 \mathrm{H}), 2.58(\mathrm{dd}, J=4.1$, $16.9,1 \mathrm{H}), 2.65-2.74(\mathrm{~m}, 1 \mathrm{H}), 3.28(\mathrm{dd}, J=7.7,16.9,1 \mathrm{H}), 7.24(\mathrm{t}, J=7.4,1 \mathrm{H}), 7.38(\mathrm{~d}$, $J=7.4,1 \mathrm{H}), 7.589 \mathrm{~d}, J=7.4,1 \mathrm{H}) ;{ }^{13} \mathrm{C} \mathrm{NMR}\left(\mathrm{CDCl}_{3}, 100 \mathrm{MHz}\right): \delta 16.4(\mathrm{q}), 17.7(\mathrm{q})$, 33.8 (t), 41.9 (d), 121.3 (d), 127.6 (d), 135.1 (d), 135.7 (s), 136.1 (s), 152.5 (s), 209.7 
(s); MS: $160\left(\mathrm{M}^{+}, 56\right), 145$ (100), 117 (19), 115 (24), 91 (11); Anal. calcd for $\mathrm{C}_{11} \mathrm{H}_{12} \mathrm{O}$ : C 82.46, H 7.55; found C 82.27, H 7.68.

\section{4-Methoxy-2-methylindan-1-one (19d)}

bp $105^{\circ} \mathrm{C}(0.025 \mathrm{mmHg})$, yield $88 \%$ from $\mathbf{6 d}, \mathrm{mp}=73-75^{\circ} \mathrm{C}$ (lit. $\left.{ }^{7} 72-73^{\circ} \mathrm{C}\right)$. Spectral data matched that reported in the literature. ${ }^{7}$

${ }^{1} \mathrm{H} \mathrm{NMR}\left(\mathrm{CDCl}_{3}, 400 \mathrm{MHz}\right): \delta 1.30(\mathrm{~d}, J=7.7,3 \mathrm{H}), 2.59(\mathrm{dd}, J=3.6 ; 17.4,1 \mathrm{H}), 2.64-$ $2.72(\mathrm{~m}, 1 \mathrm{H}), 3.31(\mathrm{dd}, J=7.7,17.4,1 \mathrm{H}), 3.89(\mathrm{~s}, 3 \mathrm{H}), 7.00-7.04(\mathrm{~m}, 1 \mathrm{H}), 7.31-7.35$ $(\mathrm{m}, 2 \mathrm{H}) ;{ }^{13} \mathrm{C} \mathrm{NMR}\left(\mathrm{CDCl}_{3}, 100 \mathrm{MHz}\right): \delta 16.4(\mathrm{q}), 31.6(\mathrm{t}), 41.8(\mathrm{~d}), 55.4(\mathrm{q}), 114.9$ (d), 115.6 (d), 128.9 (d), 137.9 (s), 142.3 (s), 157.0 (s), 209.5 (s); Anal. calcd for $\mathrm{C}_{11} \mathrm{H}_{12} \mathrm{O}_{2}$ : C 74.98, H 6.86; found C 74.91, H 6.86.

\section{References}

(1) (a) Nongkhlaw, R. L.; Nongrum, R.; Myrboh, B. J. Chem. Soc., Perkin Trans. 1 2001, 1300-1303. (b) Kraft, W. M. J. Am. Chem. Soc. 1948, 70, 3569-3571. (c) Carpenter, M. S.; Easter, W. M., Jr. 4-Tert.-Butyl $\alpha$-Methyl Hydrocinnamic Aldehyde. U.S. Patent 2875131, February 24, 1956. (d) Bogert, M. T.; Powell, G. Am. Perf. Ess. Oil Review 1930, 25, 617-620.

(2) (a) Jobashi, T.; Kawai, A.; Kawai, S.; Maeyama, K.; Oike, H.; Yoshida, Y.; Yonezawa, N. Tetrahedron 2006, 62, 5717-5724. (b) Pincock, J. A.; Young, I. S. Can. J. Chem. 2003, 81, 1083-1095.

(3) Climent, M. J.; Corma, A.; Guil-López, R.; Iborra, S.; Primo, J. J. Catal. 1998, $175,70-79$.

(4) Ichikawa, J.; Jyono, H.; Kudo, T.; Fujiwara, M.; Yokota, M. Synthesis 2005, 3946.

(5) Maillard, A.; Deluzarche, A.; El-Ass, H. C. R. Hebd. Seances Acad. Sci. 1957, $245,185-188$.

(6) Elsner, B. B.; Parker, K. J. J. Chem. Soc. 1957, 592-600.

(7) Johansson, A. M.; Mellin, C.; Hacksell, U. J. Org. Chem. 1986, 51, 5252-5258. 

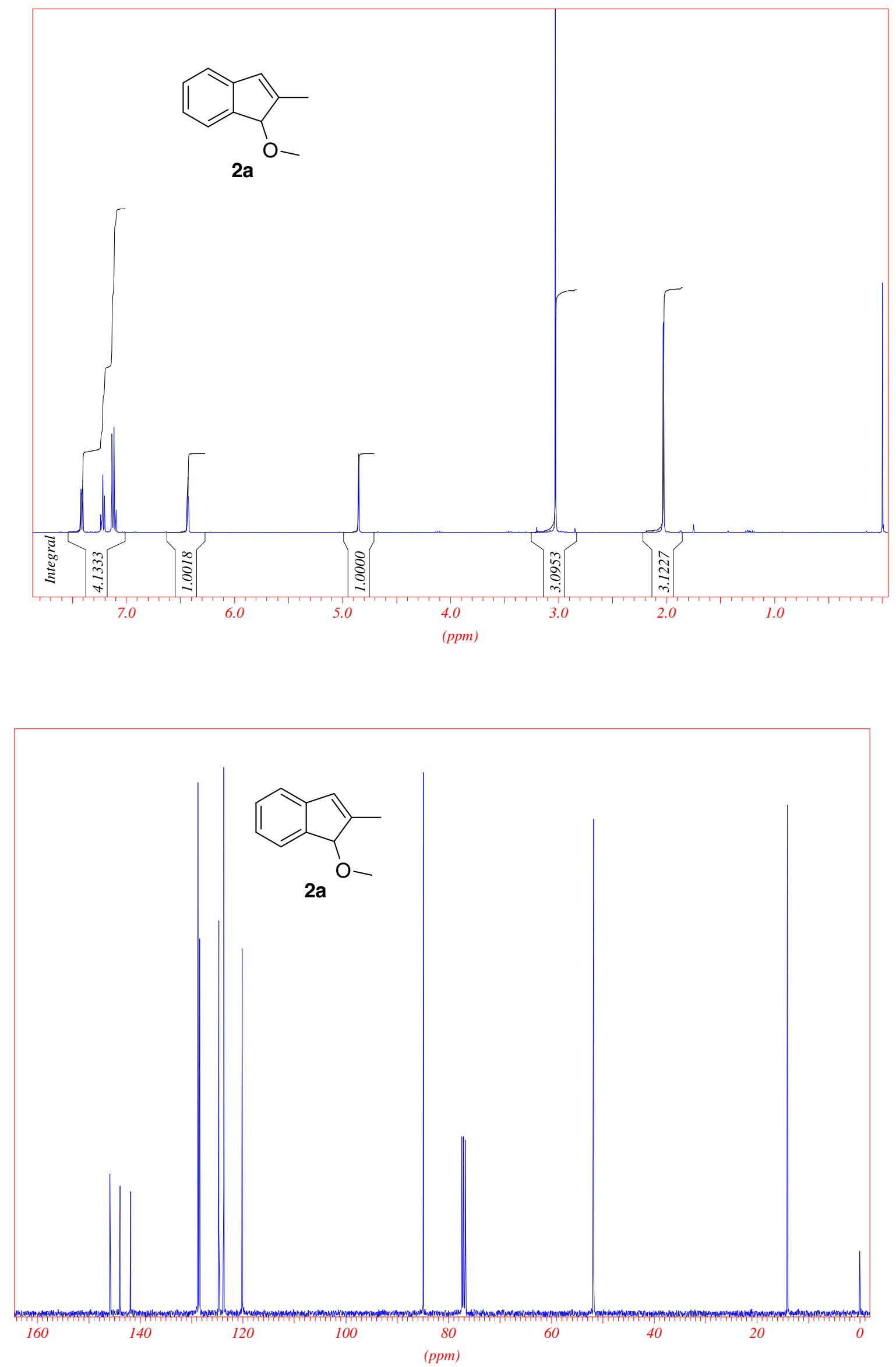

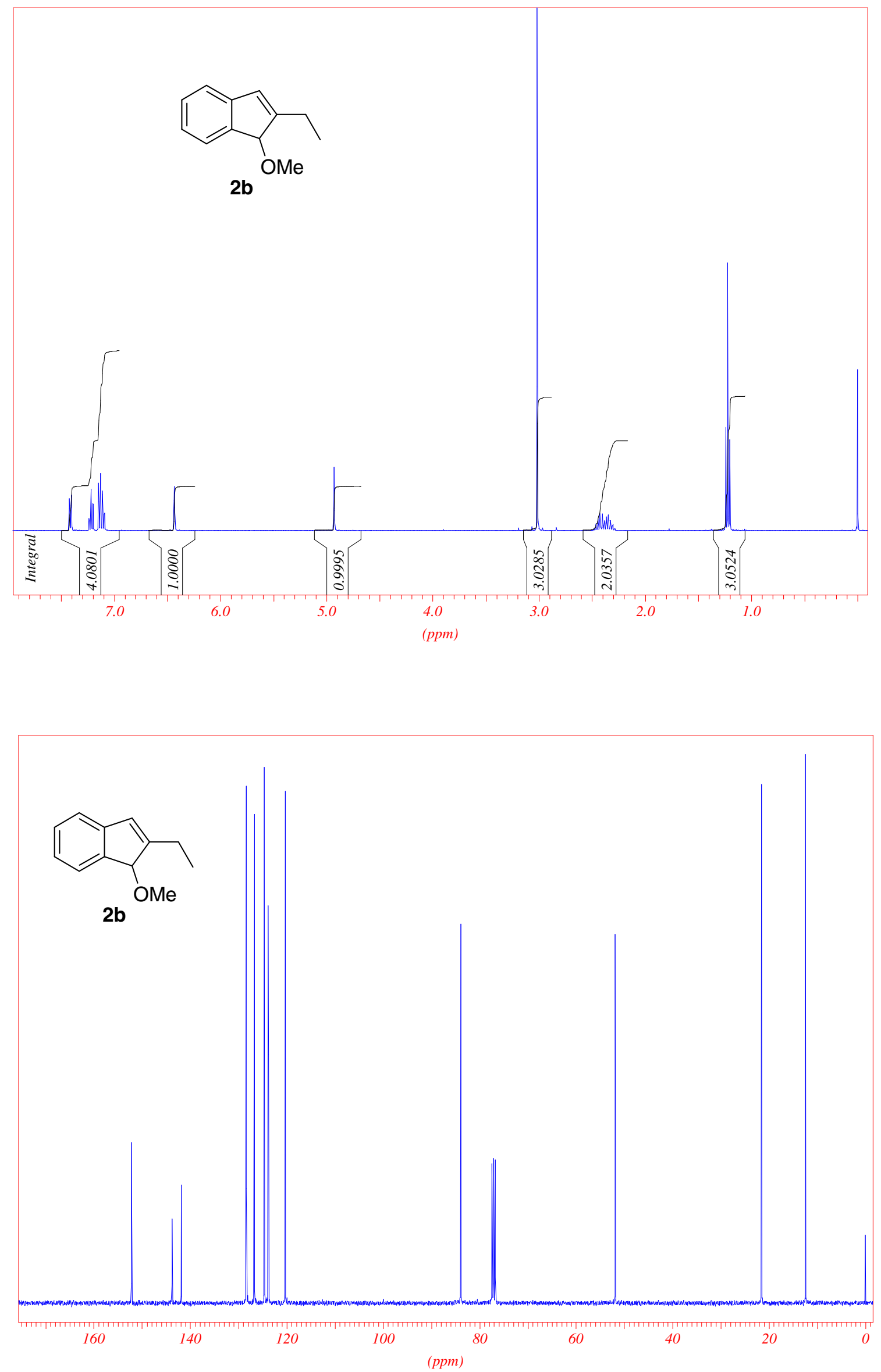

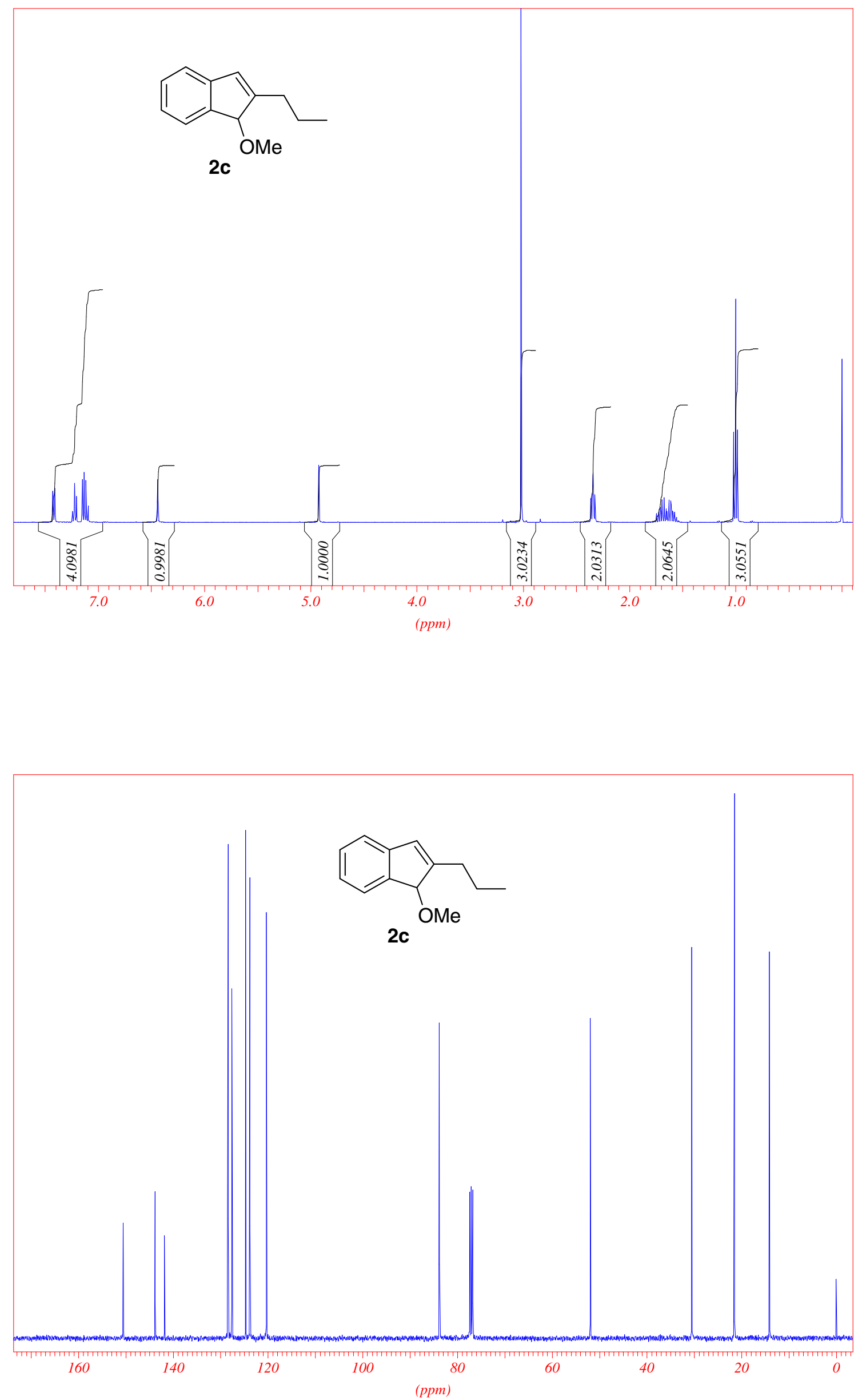

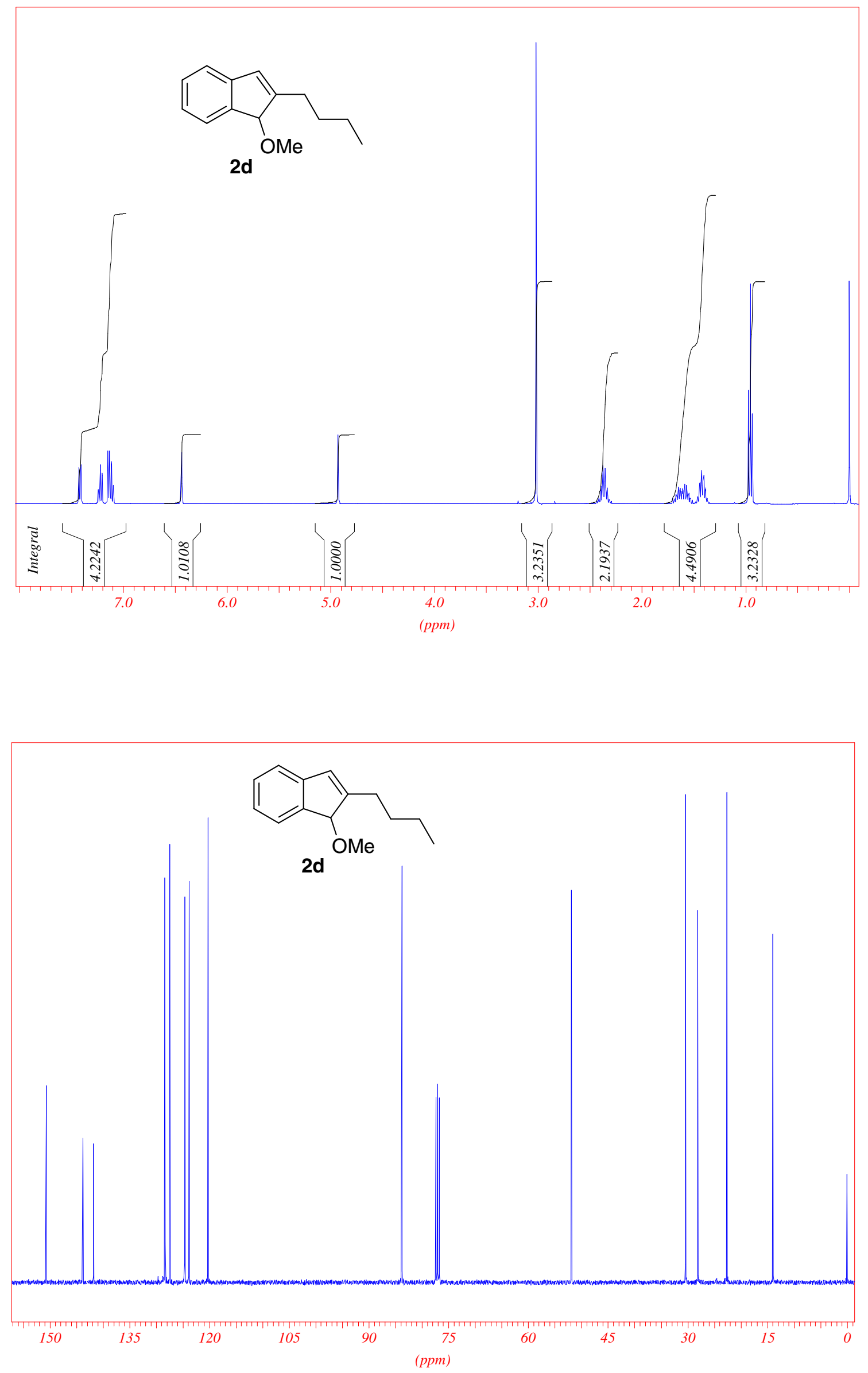

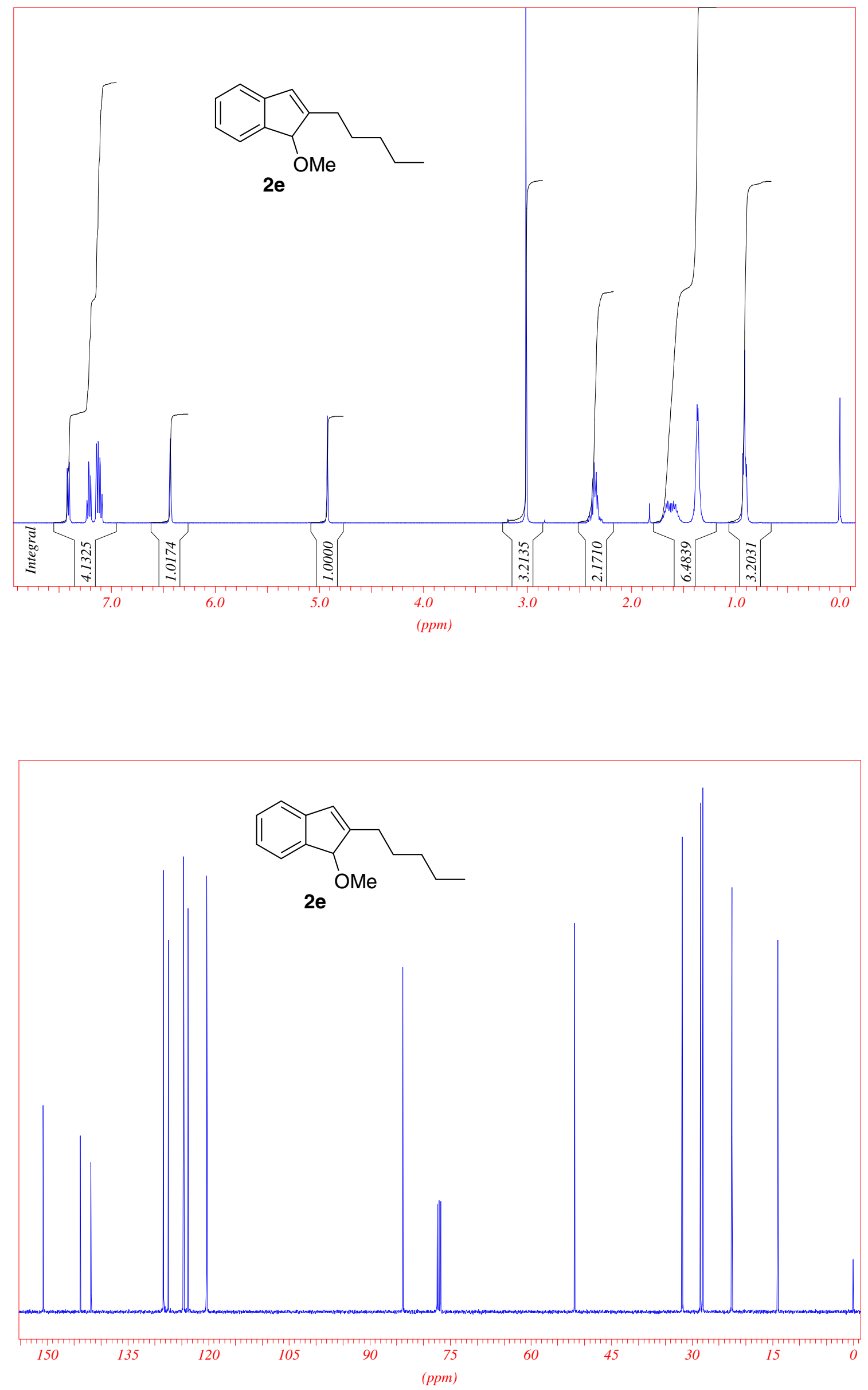

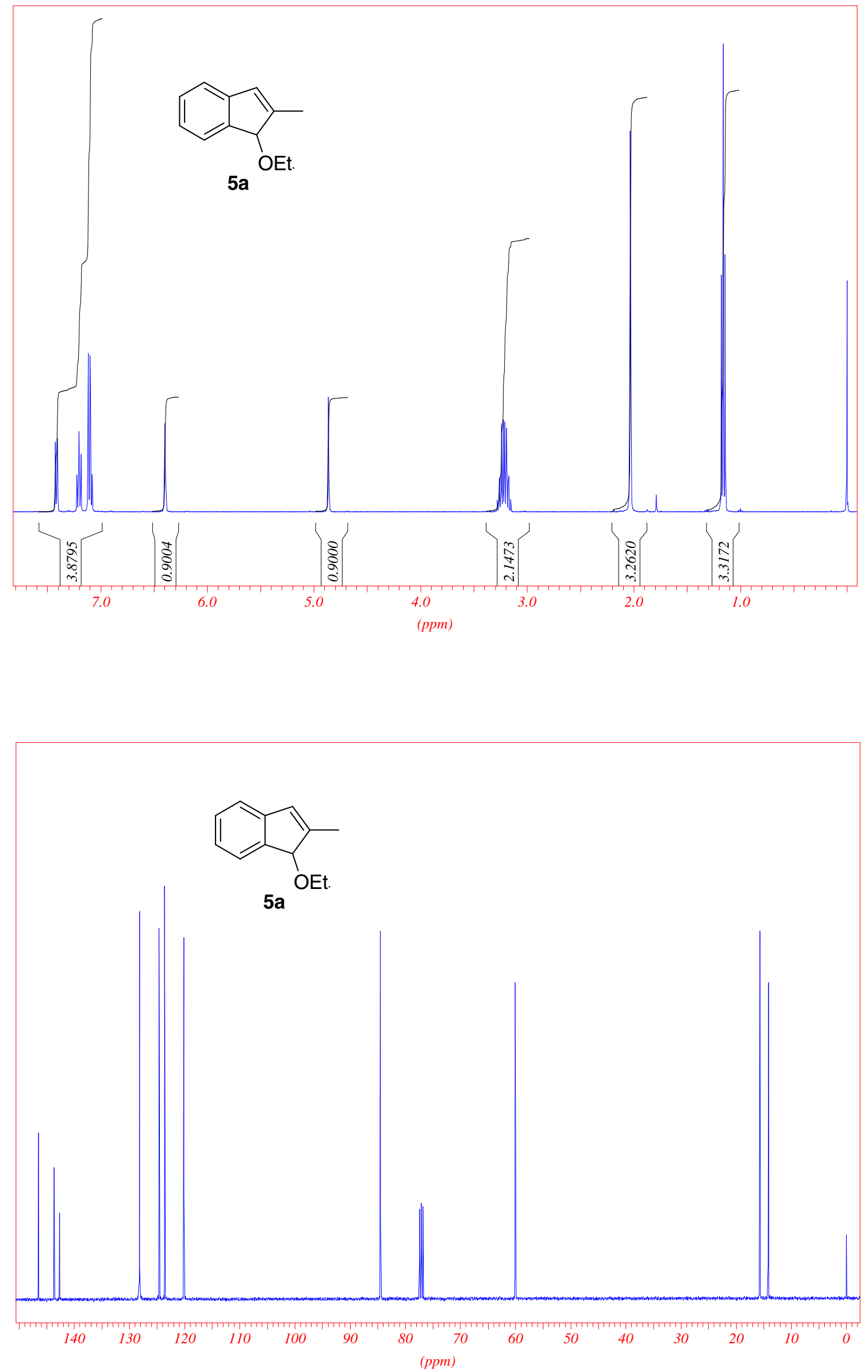

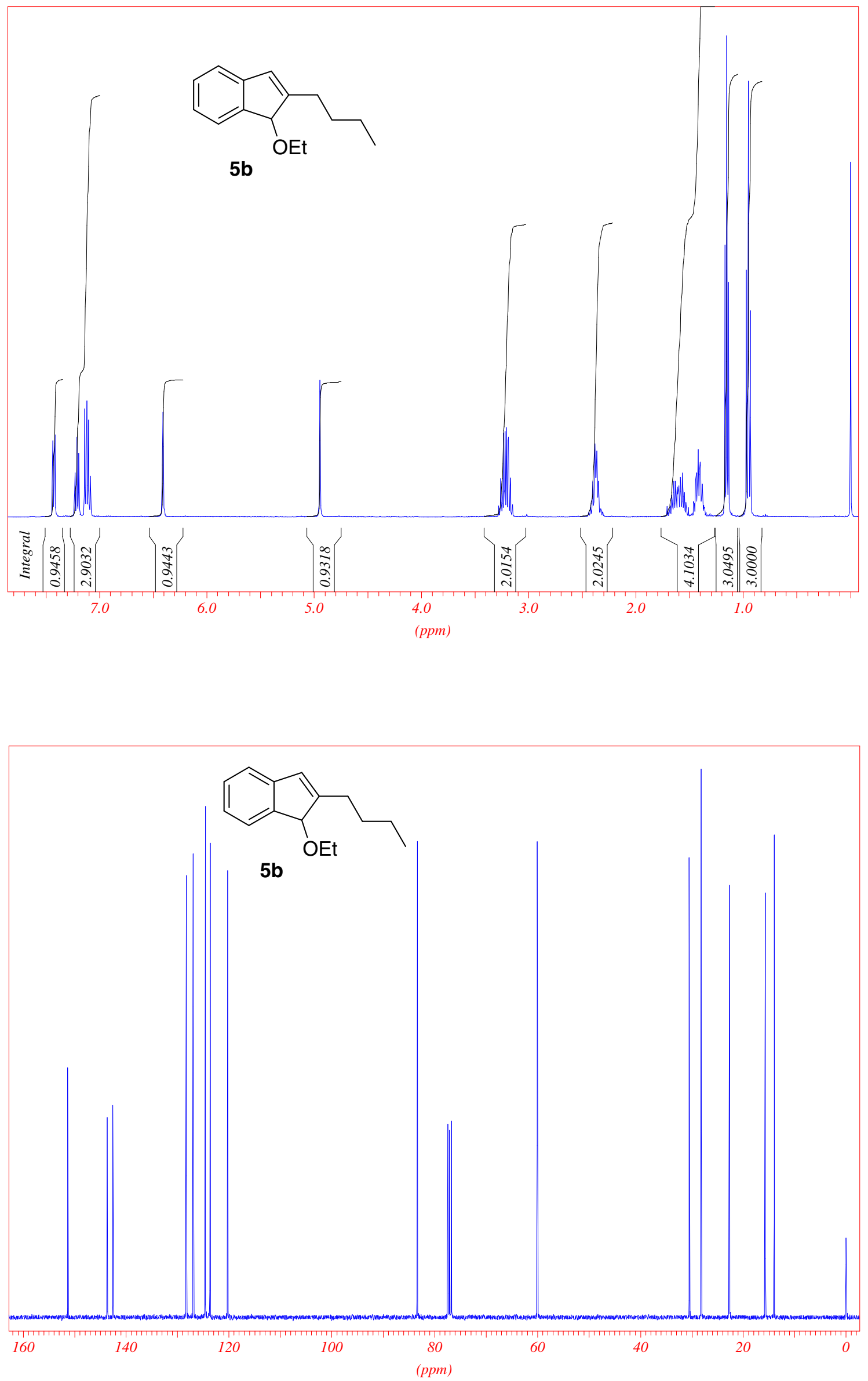

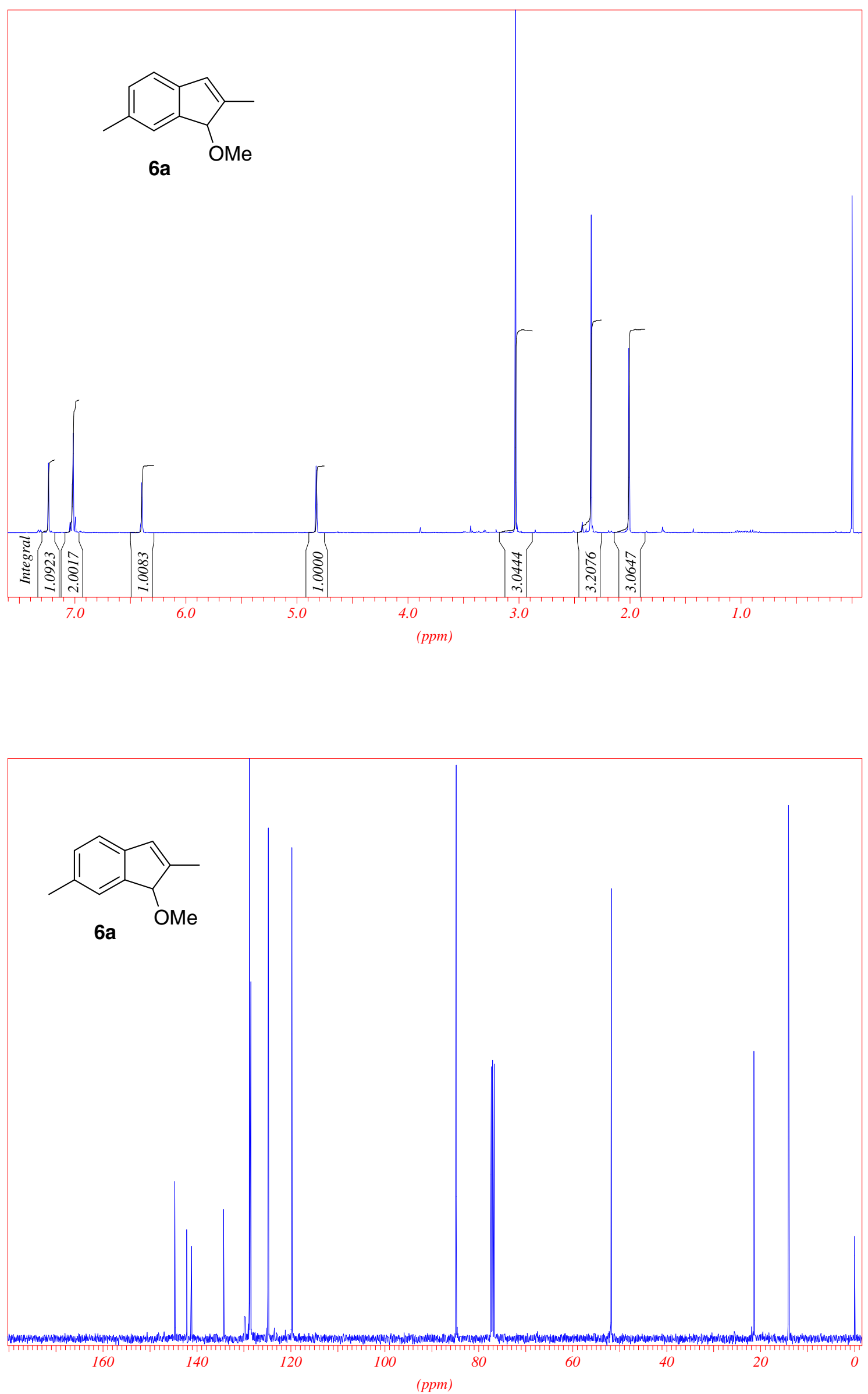

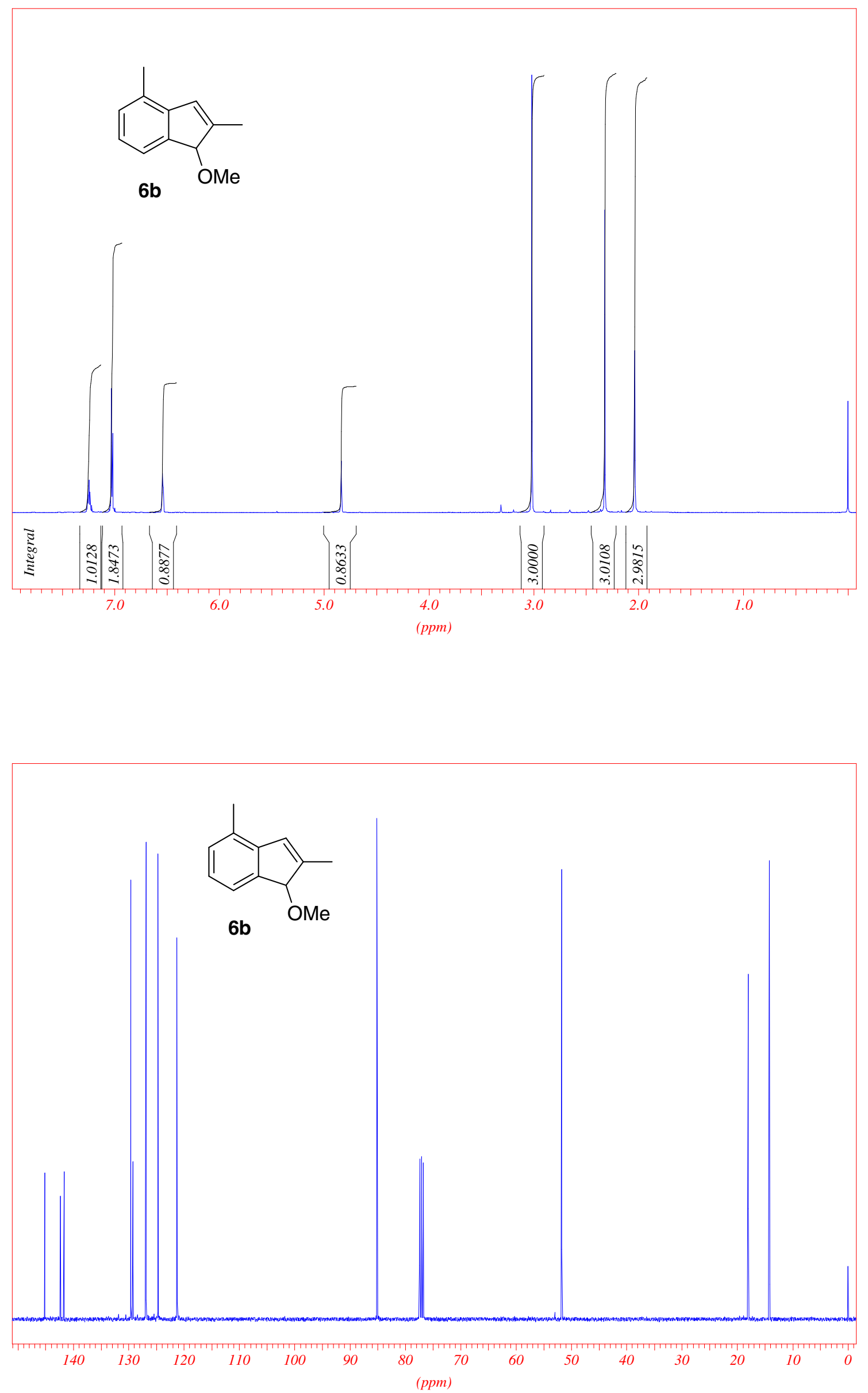

S15 

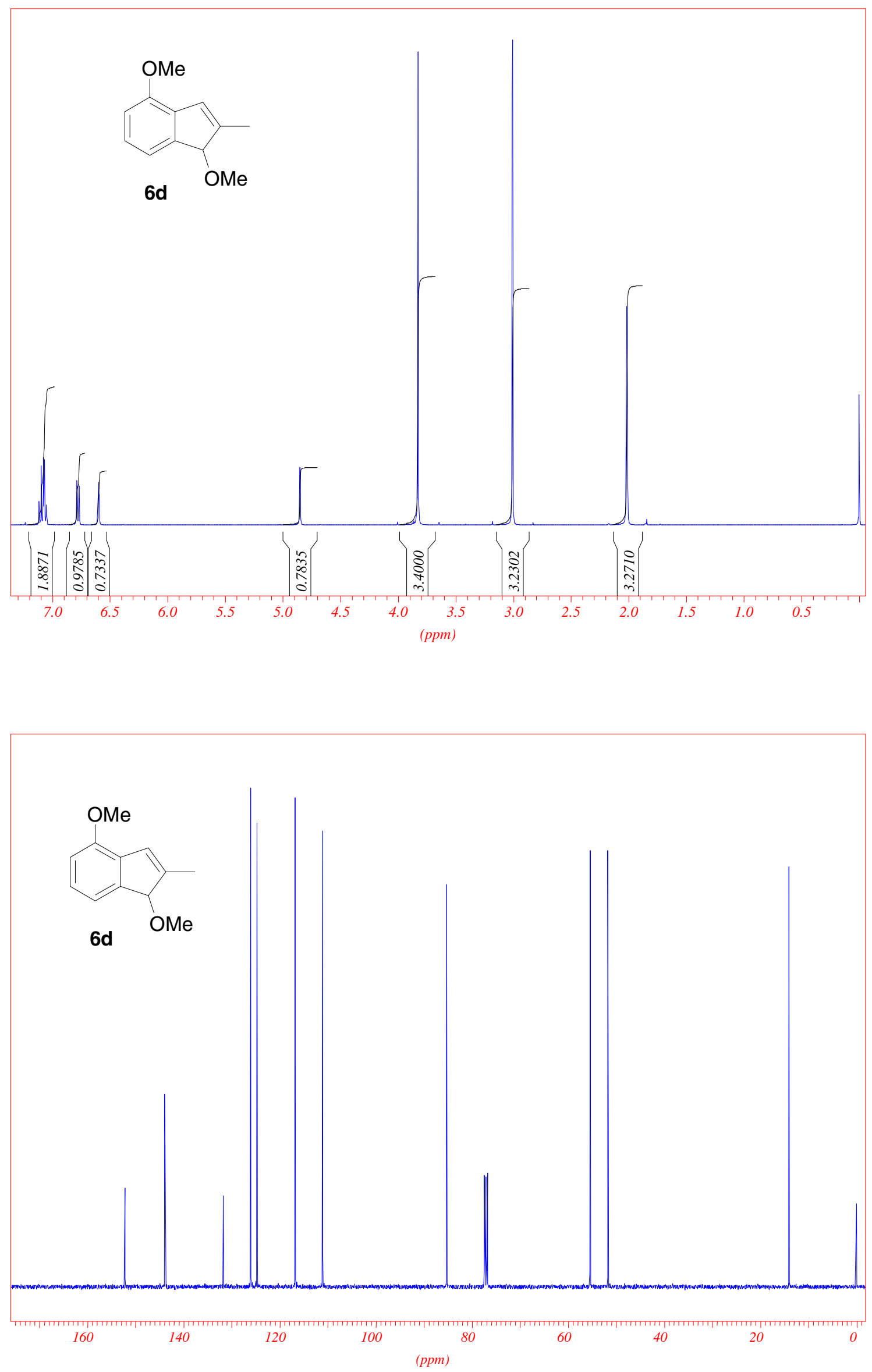

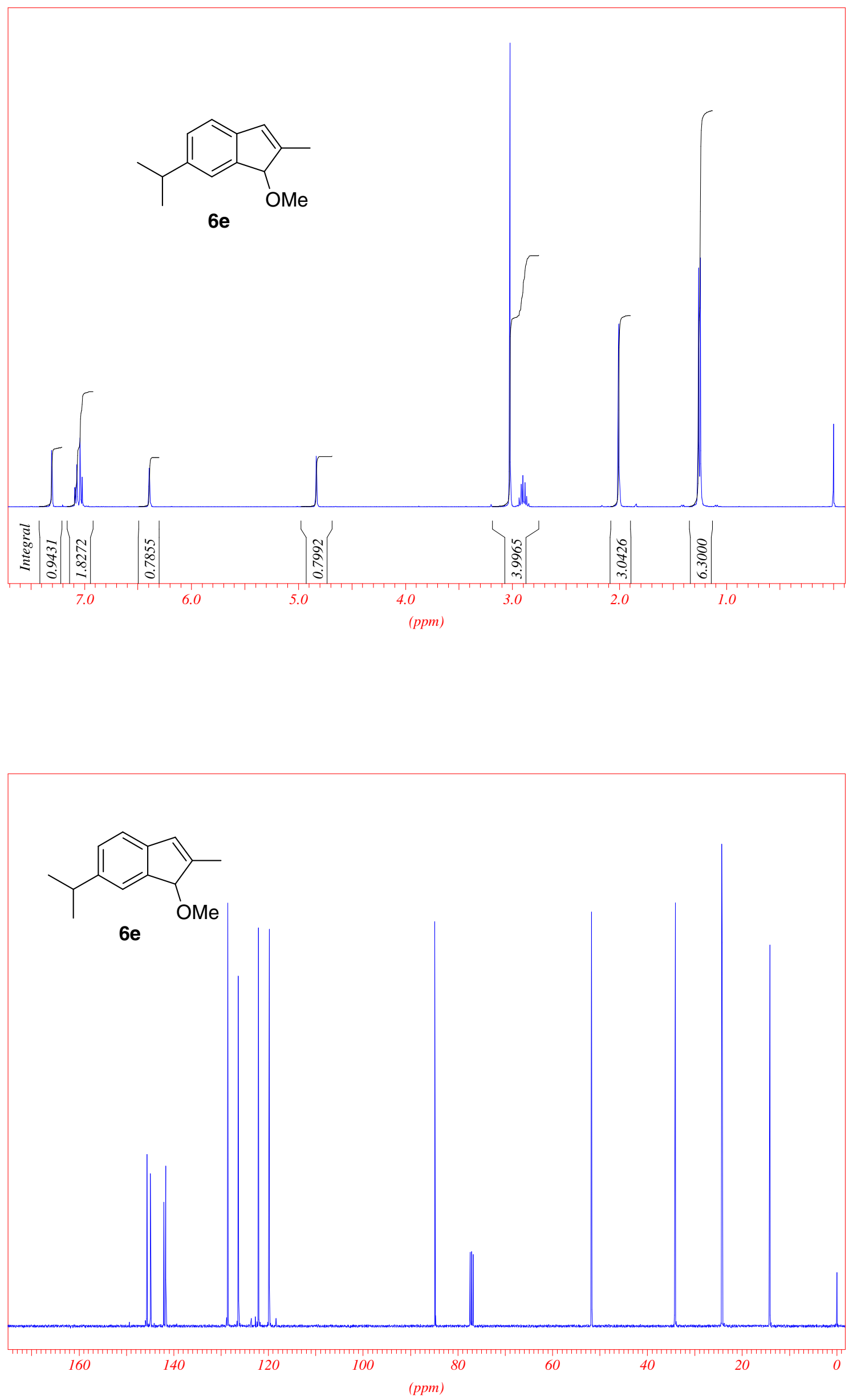

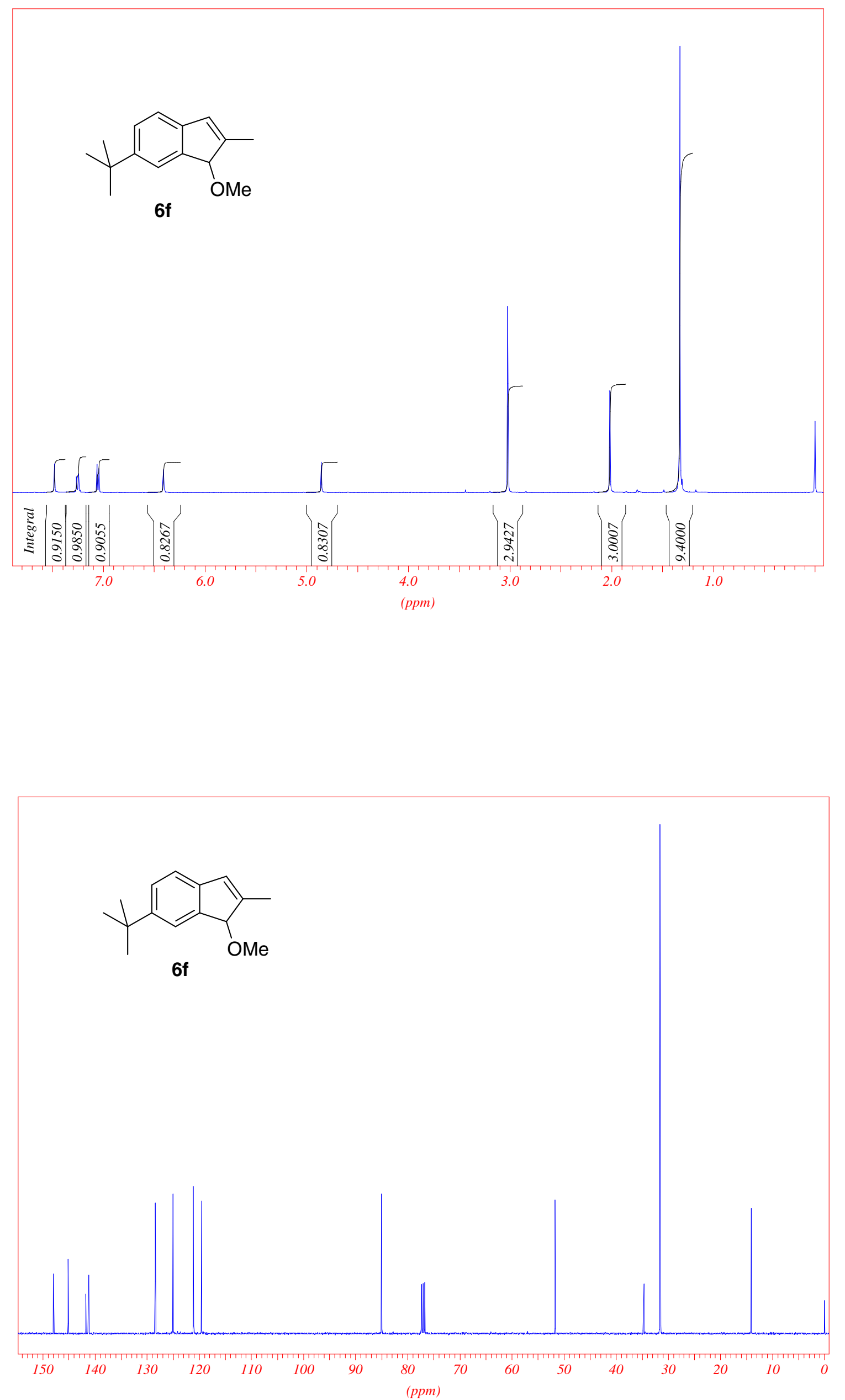

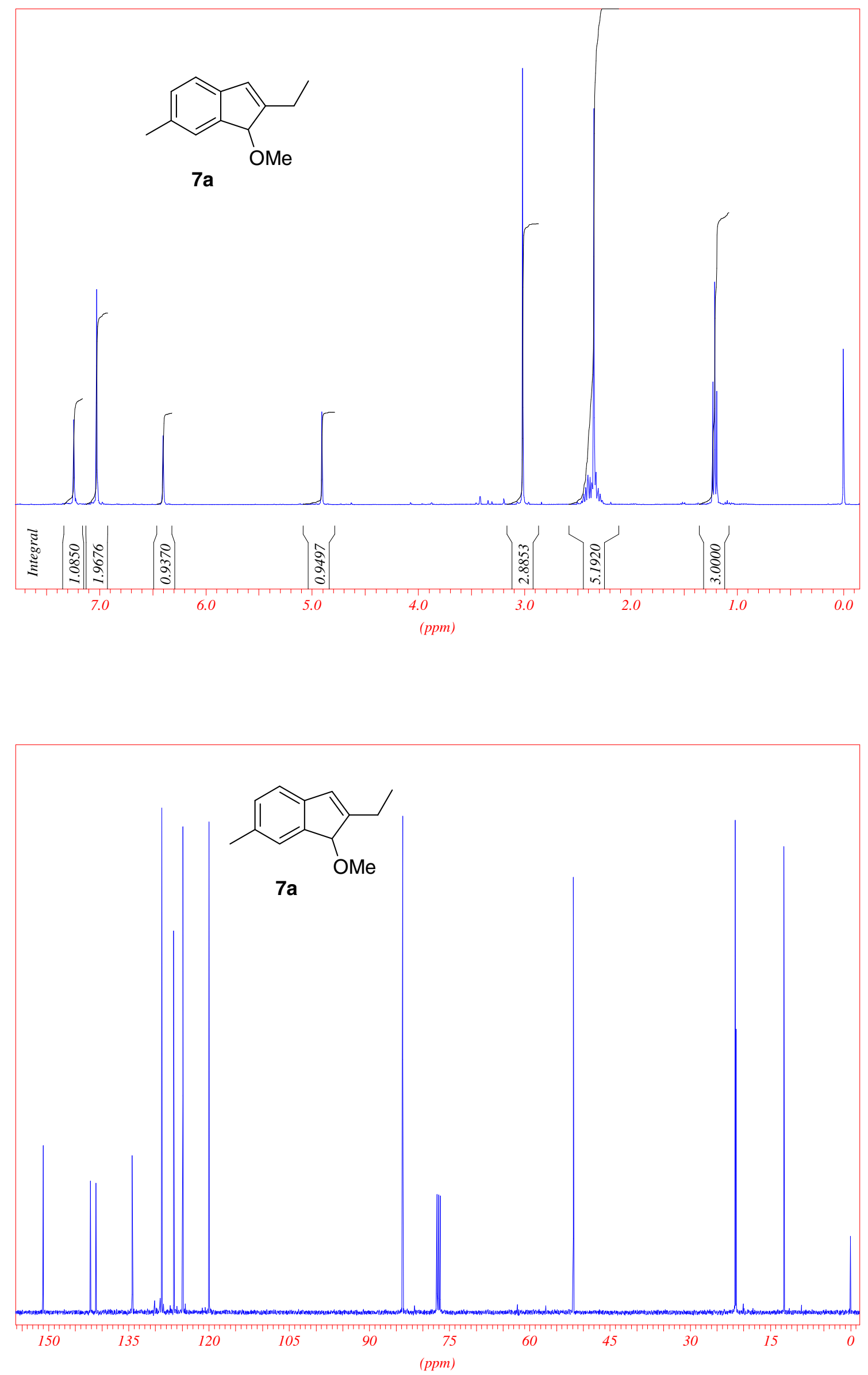

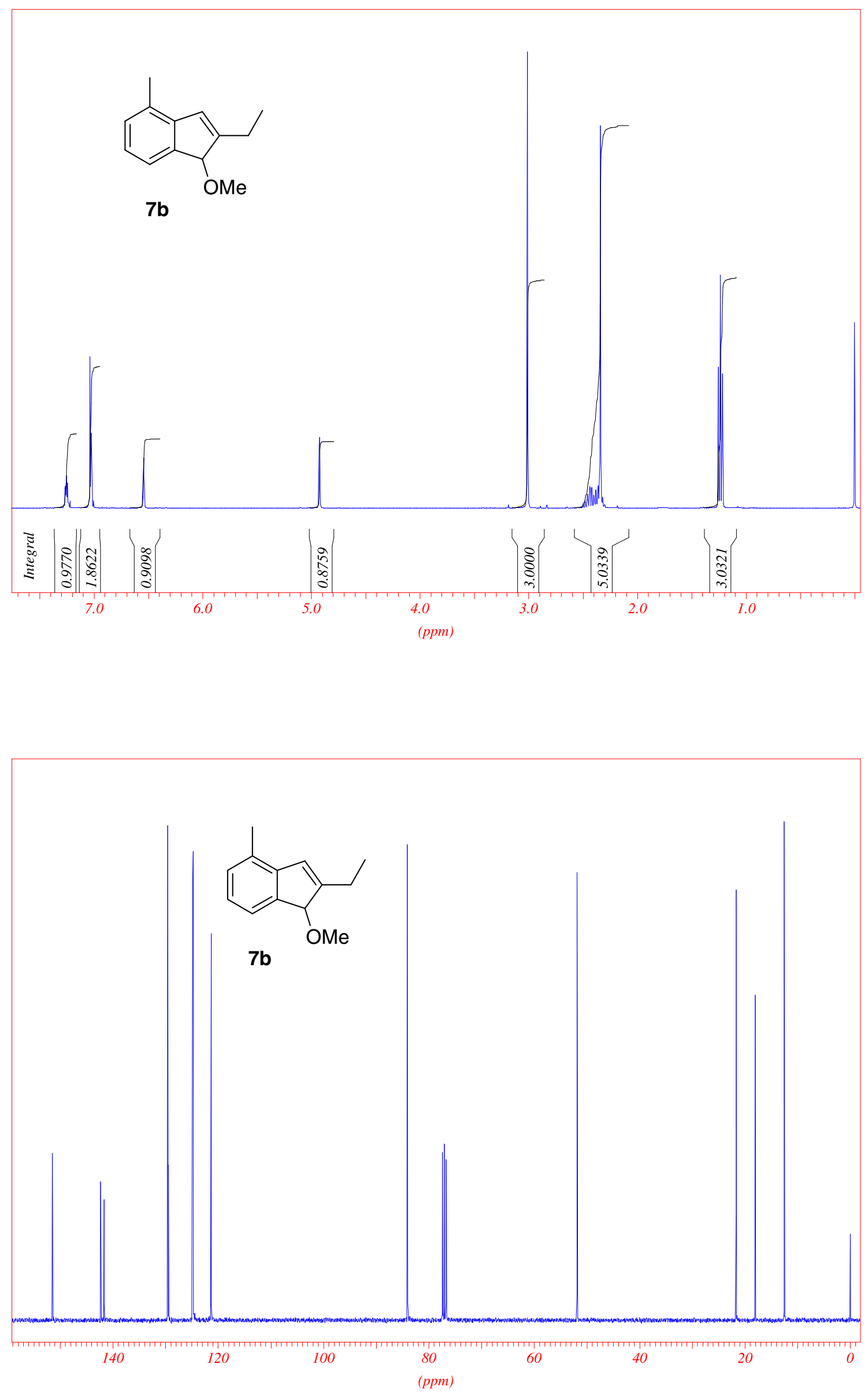

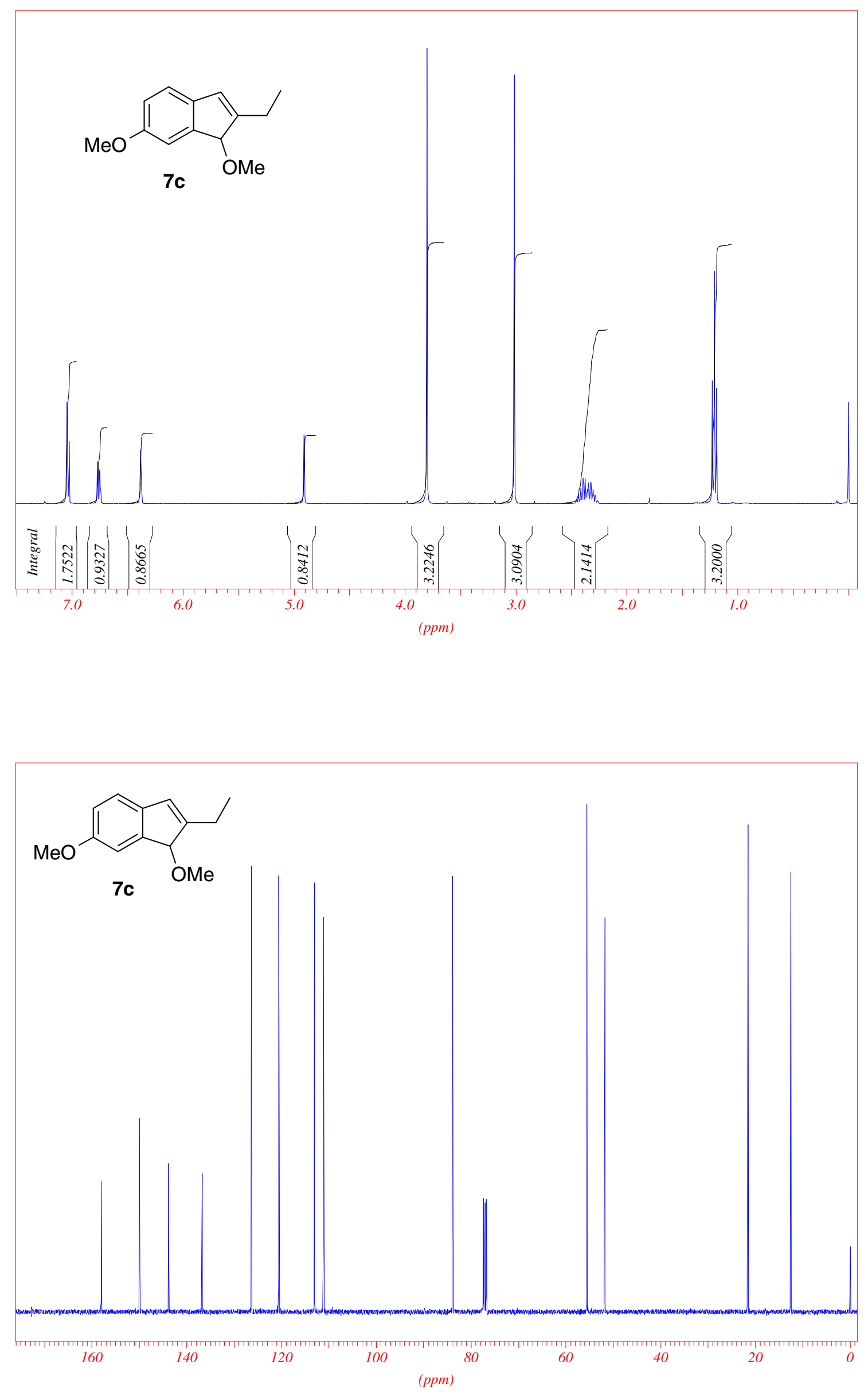

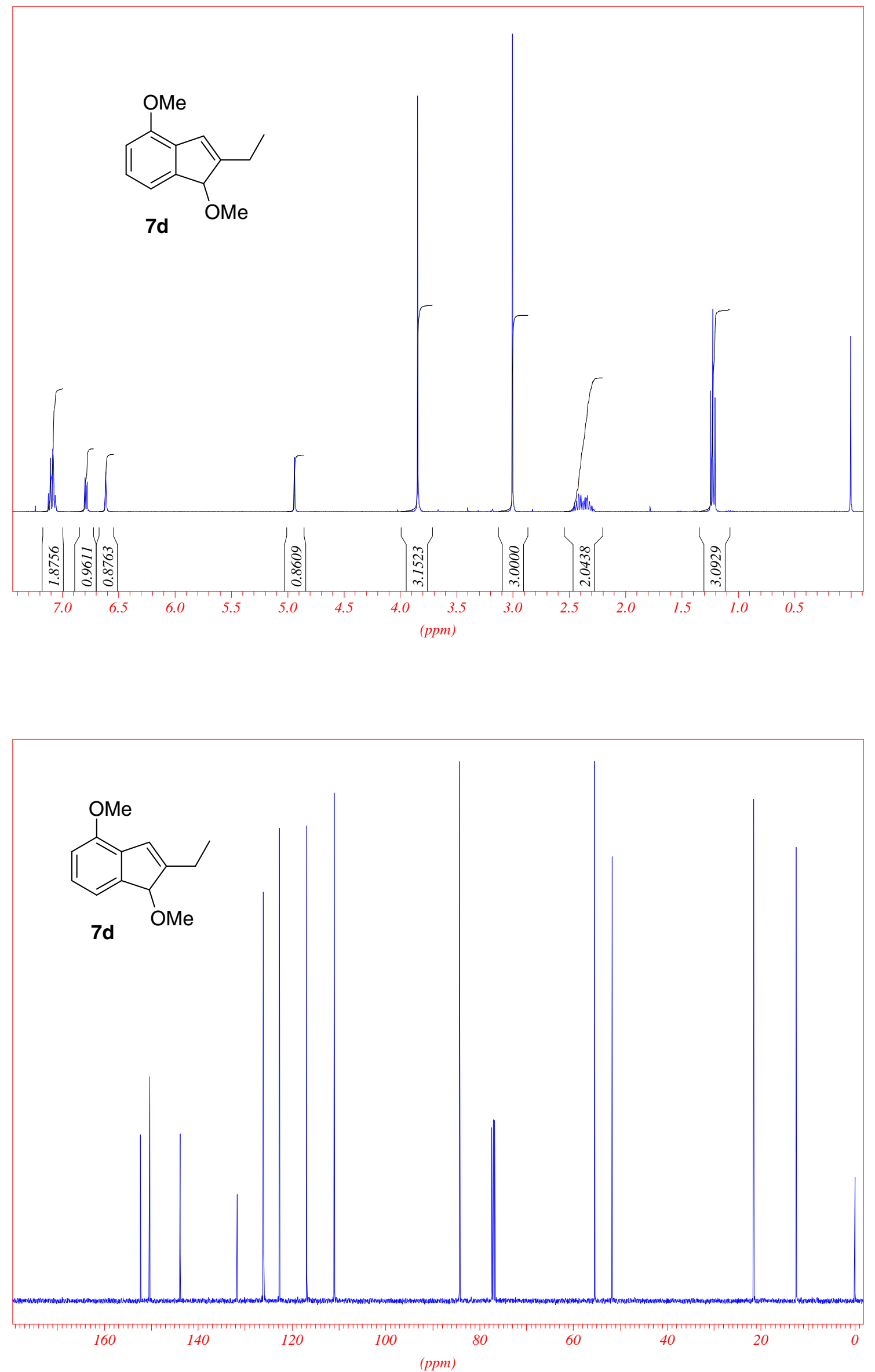

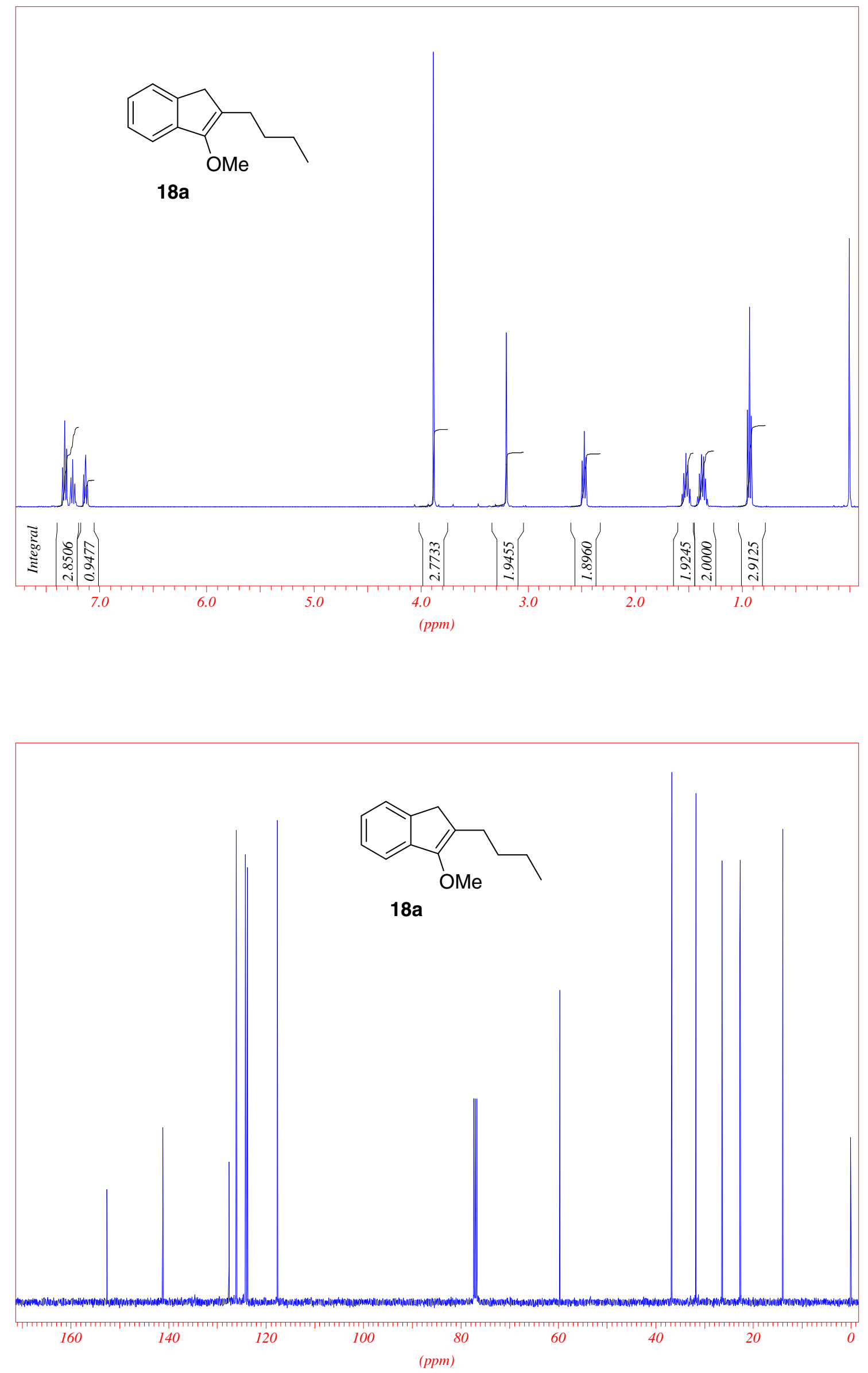

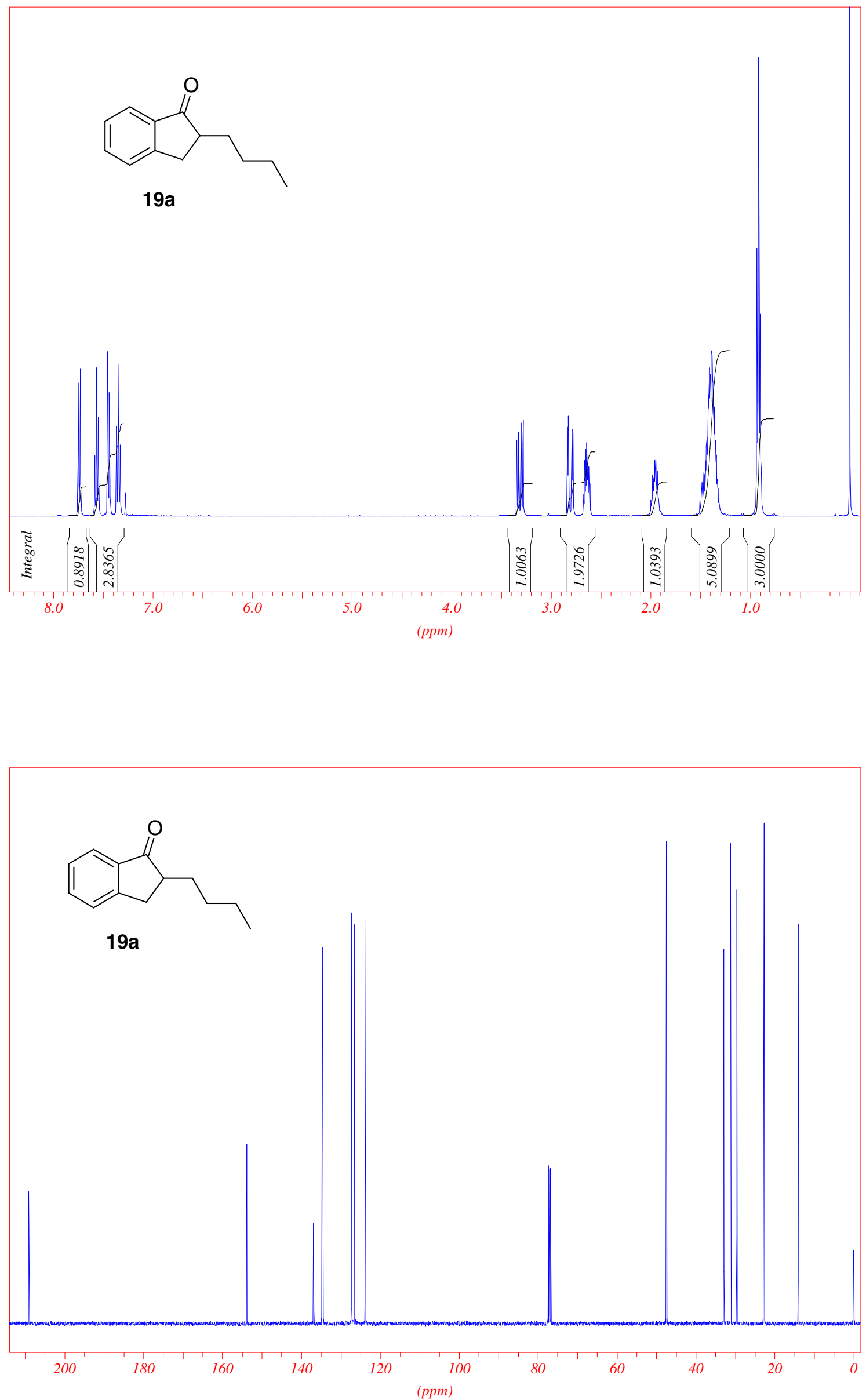

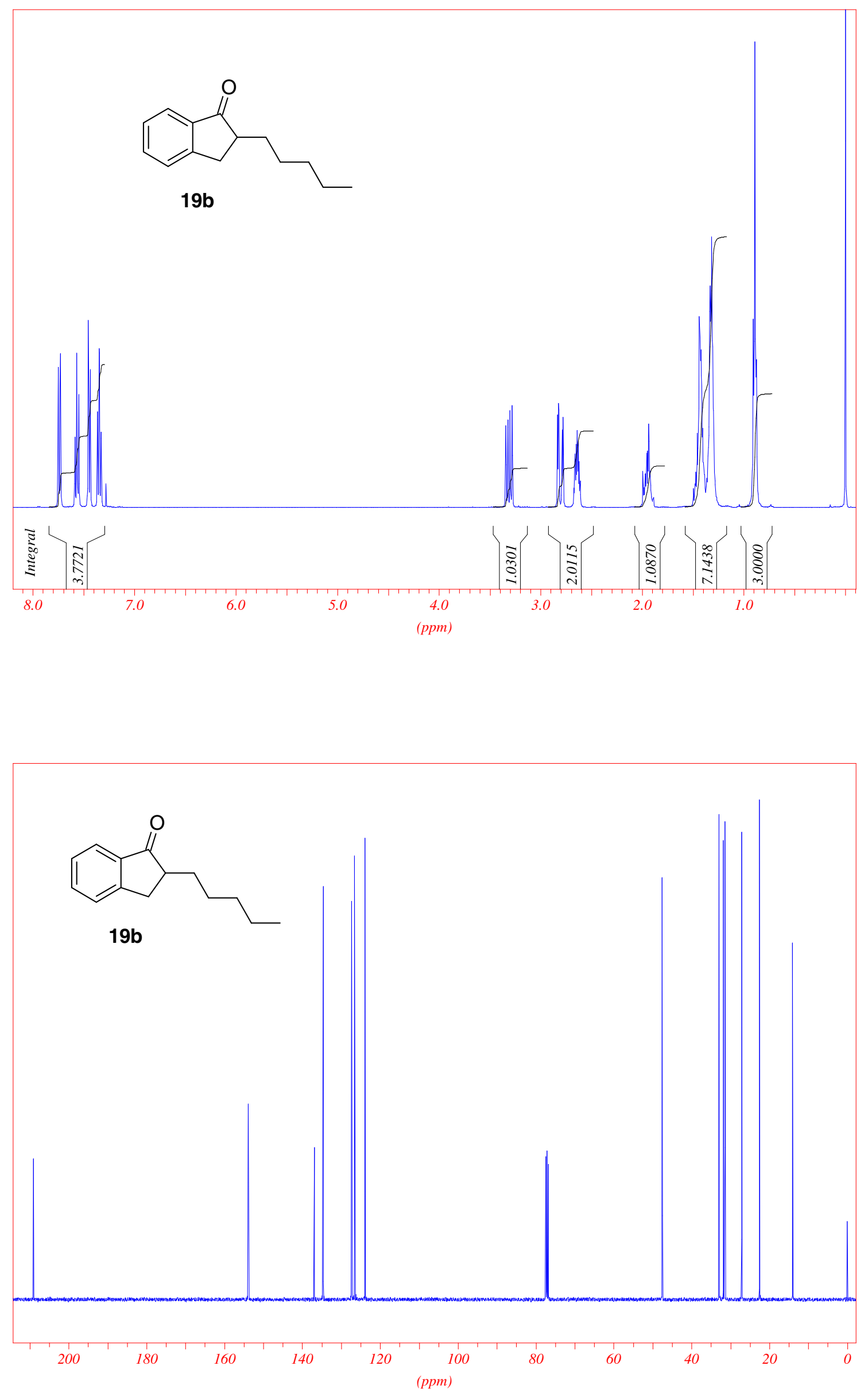

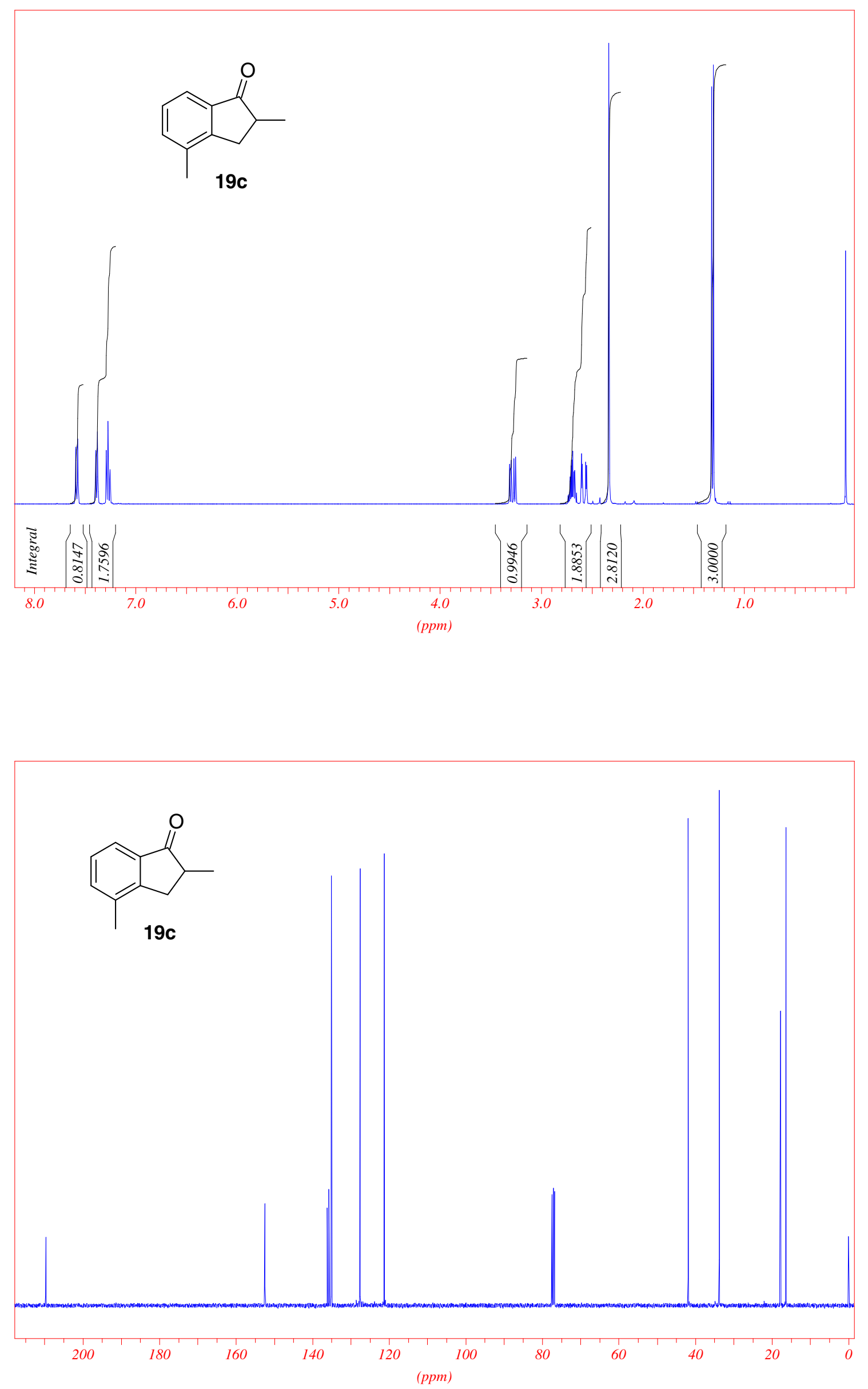

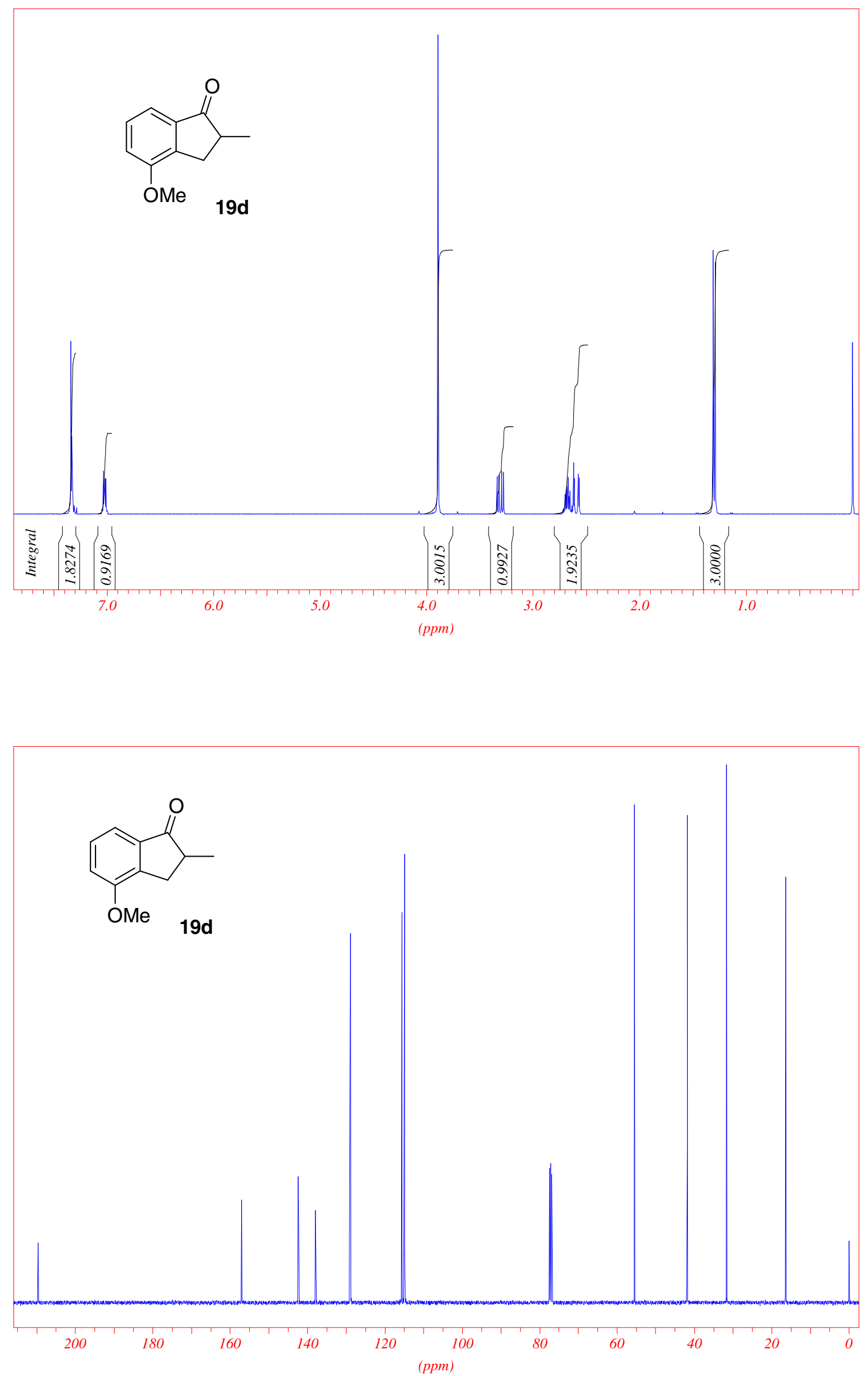